\title{
Dynamical properties of models for the Calvin cycle
}

\author{
Alan D. Rendall \\ Max Planck Institute for Gravitational Physics \\ Albert Einstein Institute \\ Am Mühlenberg 1 \\ 14476 Potsdam, Germany \\ Juan J. L. Velázquez \\ Institute for Applied Mathematics \\ University of Bonn \\ Endenicher Allee 60 \\ 53115 Bonn, Germany
}

\begin{abstract}
Modelling the Calvin cycle of photosynthesis leads to various systems of ordinary differential equations and reaction-diffusion equations. They differ by the choice of chemical substances included in the model, the choices of stoichiometric coefficients and chemical kinetics and whether or not diffusion is taken into account. This paper studies the long-time behaviour of solutions of several of these systems, concentrating on the ODE case. In some examples it is shown that there exist two positive stationary solutions. In several cases it is shown that there exist solutions where the concentrations of all substrates tend to zero at late times and others (runaway solutions) where the concentrations of all substrates increase without limit. In another case, where the concentration of ATP is explicitly included, runaway solutions are ruled out.
\end{abstract}

\section{Introduction}

Photosynthesis is a process which is of great importance for many reasons. It is the ultimate source of the food we eat, the oxygen we breath and many fuels (fossil fuels and biofuels). For this reason it is clear that it would be valuable to have a better theoretical understanding of this process and one way of approaching this task is to use mathematical models. The aim of this paper is to analyse dynamical properties of some of these models.

Photosynthesis can be split into two main parts. In the first part, called the light reactions, energy is captured from light and the small molecules ATP and 
NADPH are produced. These provide sources of energy and reducing power, respectively, for the second part, the dark reactions. The name of the latter comes from the fact that they can take place in the dark. Molecular oxygen is produced during the first part. In the second part carbon dioxide is used to make carbohydrates. For this reason this part is also known as carbon fixation. This paper is exclusively concerned with models for the second part of photosynthesis.

The models which are relevant describe reactions between different chemical substances and also, in some cases, diffusion of these chemicals. The resulting mathematical model is a system of ordinary differential equations (ODE) if diffusion is not included and a system of reaction-diffusion equations if it is. The models studied in what follows are either taken from the papers [9] or [22] or are closely related to the models in those papers. In all these cases the network of reactions modelled contains a cycle and due to the fundamental contributions of Melvin Calvin to identifying the reactions concerned this is often referred to as the Calvin cycle (see for instance [1]).

In building a model it is necessary to decide which substances are to be included. The basic unknowns are the concentrations of these substances. It is also necessary to decide how the chemical reactions are to be modelled. In most of this paper diffusion is ignored and only a few remarks are made on what happens when it is included. In the absence of diffusion the equations are of the general form

$$
\dot{x}_{i}=f_{i}(x) .
$$

Here $x_{i}$ are the concentrations, which are functions of time, and the dot denotes the time derivative. The solutions of relevance for the applications are those for which all $x_{i}$ are positive. In other words the point with coordinates $x_{i}(t)$ is always in the positive orthant $S$ of $\mathbf{R}^{n}$. The mapping $f$ with components $f_{i}$ represents the interaction between the different substances during the reactions. It is of the form $N v(x)$ where $N$ is a matrix called the stoichiometric matrix and the components $v_{\alpha}$ of $v$ are the rates of the different reactions. The $v_{\alpha}$ describe what is called the kinetics. The function $f_{i}$ is of the form $f_{i}^{+}-x_{i} f_{i}^{-}$for two nonnegative functions $f_{i}^{+}$and $f_{i}^{-}$as a result of the form of the dependence of the reaction rates on the concentrations of the substances going into the reactions. The cosets of the range of the stoichiometric matrix are called stoichiometric compatibility classes and are invariant under the flow of the dynamical system. In all the systems considered in what follows the function $v$ is $C^{1}$ and it can be shown that if the concentrations $x_{i}$ are positive at some time they remain positive as long as the solution exists. This can be proved as in the special case covered by Lemma 1 of [19. Thus $S$ is invariant under the evolution and it follows by continuity that its closure $\bar{S}$ is also invariant.

A common choice of kinetics is mass action kinetics where if $p$ molecules of the substance with concentration $x_{i}$ take part in a reaction the reaction rate has a factor proportional to $x_{i}^{p}$. This corresponds to the idea that the rate of reaction is proportional to the probability of the relevant molecules meeting. For instance in the simple reaction $A+2 B \rightarrow C$ the reaction rate is of the form $k x_{A} x_{B}^{2}$ where $k$ is the reaction constant. For more details on building systems 
of ODE describing reaction networks and mass action kinetics in particular see [7. Another common choice, particularly in the description of biological systems, is Michaelis-Menten kinetics. This is adapted to describing reactions which are dependent on a catalyst and the reactions in biological systems are usually catalysed by enzymes. If the enzymes are explicitly included in the description a type of kinetics is obtained which is referred to in 9 as MichaelisMenten represented in terms of mass action (MM-MA). Michaelis-Menten (MM) kinetics is obtained from MM-MA kinetics by a limiting process (quasistationary approximation).

The structure of the paper is as follows. In Section 2 the dynamics of models with mass action kinetics is considered for two different choices of the stoichiometric coefficients. In particular it is shown that for certain values of the reaction constants there is exactly one positive steady state and that it is unstable. This raises the question of the final fate of general solutions. It turns out that for suitable choices of the reaction constants there is an open set of initial data for which all concentrations tend to zero at late times and an open set of initial data for which all concentrations tend to infinity at late times. The main results are collected in Theorem 1. The second statement is somewhat technical to prove for the choice of stoichiometric coefficients used in [9] and the proof is the subject of Section 3 . Section 4 is concerned with the models where the kinetics is Michaelis-Menten represented in terms of mass action. It is shown that there are solutions which tend to infinity at late times for both the choices of stoichiometric coefficients made in [9] and in 22. For the first case it is proved that there can exist more than one positive stationary solution in a stoichiometric compatibility class and there is some discussion of what happens in the second case. The models with Michaelis-Menten kinetics are studied in Section 5 and it is shown that that there are solutions which tend to infinity at late times for that model too. It is shown that the stationary solutions are essentially the same for the MM-MA and MM models. A model in which the concentration of ATP is a dynamical variable is discussed in Section 6. This leads to a system of ODE for which, in contrast to the models discussed up to this point, all solutions are bounded in the future. In all this the aim is to treat values of the reaction constants which are as general as possible. In Section 7 some conclusions are drawn. Appendix A gives an introduction to Michaelis-Menten theory. Appendix B collects some technical results required for the proofs in the main text.

\section{Mass action kinetics}

This section is mainly concerned with the dynamical system (6) of [9]. There are five variables $x_{\mathrm{RuBP}}, x_{\mathrm{PGA}}, x_{\mathrm{DPGA}}, x_{\mathrm{GAP}}$ and $x_{\mathrm{Ru} 5 \mathrm{P}}$ which are the concentrations of the substances abbreviated by the subscripts. They are ribulose1,5-bisphosphate (RuBP), 3-phosphoglycerate (PGA), 1,3-diphosphoglycerate (DPGA), glyceraldehyde-3-phosphate (GAP) and ribulose-5-phosphate (Ru5P). This system has mass action kinetics and is called MA in what follows. It is 
given by

$$
\begin{aligned}
& \frac{d x_{\mathrm{RuBP}}}{d t}=k_{5} x_{\mathrm{Ru} 5 \mathrm{P}}-k_{1} x_{\mathrm{RuBP}}, \\
& \frac{d x_{\mathrm{PGA}}}{d t}=2 k_{1} x_{\mathrm{RuBP}}-k_{2} x_{\mathrm{PGA}}-k_{6} x_{\mathrm{PGA}}, \\
& \frac{d x_{\mathrm{DPGA}}}{d t}=k_{2} x_{\mathrm{PGA}}-k_{3} x_{\mathrm{DPGA}}, \\
& \frac{d x_{\mathrm{GAP}}}{d t}=k_{3} x_{\mathrm{DPGA}}-5 k_{4} x_{\mathrm{GAP}}^{5}-k_{7} x_{\mathrm{GAP}}, \\
& \frac{d x_{\mathrm{Ru} 5 \mathrm{P}}}{d t}=-k_{5} x_{\mathrm{Ru} 5 \mathrm{P}}+3 k_{4} x_{\mathrm{GAP}}^{5} .
\end{aligned}
$$

The $k_{i}$ are the reaction constants and they are all positive. The alternative notation where $\left(x_{\mathrm{RuBP}}, x_{\mathrm{PGA}}, x_{\mathrm{DPGA}}, x_{\mathrm{GAP}}, x_{\mathrm{Ru} 5 \mathrm{P}}\right)$ is replaced by $\left(x_{1}, x_{2}, x_{3}, x_{4}, x_{5}\right)$ is also used. The state space of interest for the applications is the positive orthant $S$. Sometimes it is also useful to consider the dynamics on $\bar{S}$. The origin is a stationary solution. The linearization of the system at the origin has eigenvalues $\left(-k_{1},-k_{2}-k_{6},-k_{3},-k_{7},-k_{5}\right)$. Thus the origin is a hyperbolic sink. Consider a solution which starts at a point of the boundary of $S$ other than the origin. Let $N$ be the set of indices $i$ for which the concentration $x_{i}$ vanishes. Both $N$ and its complement are non-empty. Hence there exists $i \notin N$ for which $j \in N$ for $j=i+1 \bmod 5$. It follows that $\dot{x}_{j}>0$ and so the extension of the solution towards the past must lie in the complement of $\bar{S}$. This implies that there exists no solution other than the zero solution which stays in the boundary of $S$ for a finite time. This can be used to show that if $x$ is a solution which starts in $S$ then its $\omega$-limit set contains no point of the boundary of $S$ other than the origin. For suppose that $x^{*}$ is a point of the $\omega$-limit set of $x(t)$ which belongs to the boundary of $S$ and is not the origin. Then there is a solution $y$ which passes through $x^{*}$ and lies entirely in the $\omega$-limit set of $x$ and hence in $\bar{S}$. On the other hand it has just been shown that this cannot happen. Thus any $\omega$-limit point of a solution starting in $S$ must either be the origin or a point of $S$.

Taking a suitable linear combination of (2.4) and (2.5) eliminates the nonlinear terms.

$$
\frac{d\left(3 x_{\mathrm{GAP}}+5 x_{\mathrm{Ru} 5 \mathrm{P}}\right)}{d t}=3 k_{3} x_{\mathrm{DPGA}}-3 k_{7} x_{\mathrm{GAP}}-5 k_{5} x_{\mathrm{Ru} 5 \mathrm{P}} .
$$

Let $X$ be the maximum of the quantities $x_{\mathrm{RuBP}}, x_{\mathrm{PGA}}, x_{\mathrm{DPGA}}, x_{\mathrm{GAP}}$ and $3 x_{\mathrm{GAP}}+5 x_{\mathrm{Ru} 5 \mathrm{P}}$. Then any solution of the system satisfies the integral inequality

$$
X(t) \leq X\left(t_{0}\right)+C \int_{t_{0}}^{t} X(s) d s
$$

where $C$ is the maximum of $2 k_{1}, k_{2}, 3 k_{3}$ and $\frac{1}{5} k_{5}$. Thus, by Gronwall's inequality, none of the variables can blow up in finite time. Together with the fact 
that $\bar{S}$ is invariant this shows that the solution exists globally in the future. Summing up what has been proved so far gives:

Proposition 1 A solution of (2.1)-(2.5) with positive initial data exists globally to the future, remains positive and has no $\omega$-limit points on the boundary of $S$ except possibly the origin.

It is shown in 9] that if $k_{2}>5 k_{6}$ there is a unique stationary solution of the system in $S$ and the equilbrium concentrations are calculated explicitly in terms of the reaction constants. For this solution

$$
x_{\mathrm{RuBP}}=k_{1}^{-1}\left[\frac{k_{7}^{5}}{3 k_{4}\left(\frac{2 k_{2}}{k_{2}+k_{6}}-\frac{5}{3}\right)^{5}}\right]^{\frac{1}{4}} \text {. }
$$

The other equilibrium concentrations can be expressed as $x_{\mathrm{Ru} 5 \mathrm{P}}=\frac{k_{1}}{k_{5}} x_{\mathrm{RuBP}}$, $x_{\mathrm{PGA}}=\frac{2 k_{1}}{k_{2}+k_{6}} x_{\mathrm{RuBP}}, x_{\mathrm{DPGA}}=\frac{2 k_{1} k_{2}}{k_{3}\left(k_{2}+k_{6}\right)} x_{\mathrm{RuBP}}, x_{\mathrm{GAP}}=\left(\frac{k_{1}}{3 k_{4}} x_{\mathrm{RuBP}}\right)^{\frac{1}{5}} . \mathrm{An}$ additional relation which can be derived for the stationary solution is that $x_{\mathrm{GAP}}^{4}=\frac{k_{7}\left(k_{2}+k_{6}\right)}{k_{4}\left(k_{2}-5 k_{6}\right)}$. The linearization of the system about the stationary solution has the characteristic polynomial

$$
\left(\lambda+k_{1}\right)\left(\lambda+k_{2}+k_{6}\right)\left(\lambda+k_{3}\right)\left(\lambda+25 k_{4} x_{\mathrm{GAP}}^{4}+k_{7}\right)\left(\lambda+k_{5}\right)-30 k_{1} k_{2} k_{3} k_{4} k_{5} x_{\mathrm{GAP}}^{4} .
$$

The constant term is equal to

$$
k_{1} k_{3} k_{5}\left[\left(k_{2}+k_{6}\right) k_{7}+5\left(-k_{2}+5 k_{6}\right) k_{4} x_{\mathrm{GAP}}^{4}\right]=-4 k_{1}\left(k_{2}+k_{6}\right) k_{3} k_{5} k_{7} .
$$

Because of the signs of the coefficients this polynomial has exactly one positive root. This means in particular that the stationary solution is unstable. For $k_{2} \leq 5 k_{6}$ there is no stationary solution in $S$. If $k_{2}-5 k_{6}$ is allowed to tend to zero while each of the reaction constants tends to a non-zero value then the stationary point tends to infinity.

Define a function

$$
L_{1}=x_{\mathrm{RuBP}}+\frac{1}{2} x_{\mathrm{PGA}}+\frac{3}{5} x_{\mathrm{DPGA}}+\frac{3}{5} x_{\mathrm{GAP}}+x_{\mathrm{Ru} 5 \mathrm{P}} .
$$

Then

$$
\frac{d L_{1}}{d t}=-\frac{1}{2}\left(k_{6}-\frac{1}{5} k_{2}\right) x_{\mathrm{PGA}}-\frac{3}{5} k_{7} x_{\mathrm{GAP}}
$$

If $k_{2} \leq 5 k_{6}$ then $L_{1}$ is a Lyapunov function for the system (2.1)-(2.5). This recovers the fact that for this parameter range there are no stationary solutions. It fact, when combined with Proposition 1, it shows that when that inequality holds all solutions converge to the origin as $t \rightarrow \infty$ - all concentrations go to zero at late times. Thus strong control of the late-time asymptotics of all solutions has been obtained in this case.

It remains to consider the case $k_{2}>5 k_{6}$ where $L_{1}$ does not seem to give an interesting conclusion. A useful generalization of $L_{1}$ is given by

$$
L_{2}=x_{\mathrm{RuBP}}+\frac{1}{2} x_{\mathrm{PGA}}+\alpha x_{\mathrm{DPGA}}+\alpha x_{\mathrm{GAP}}+x_{\mathrm{Ru} 5 \mathrm{P}}
$$


for $\alpha>0$. It satisfies

$$
\frac{d L_{2}}{d t}=-\frac{1}{2}\left[k_{6}-(2 \alpha-1) k_{2}\right] x_{\mathrm{PGA}}-\left[\alpha k_{7}-(3-5 \alpha) k_{4} x_{\mathrm{GAP}}^{4}\right] x_{\mathrm{GAP}} .
$$

This means that if $(2 \alpha-1) k_{2} \leq k_{6}$ the function $L_{2}$ is monotone decreasing along any solution for which $(3-5 \alpha) k_{4} x_{\mathrm{GAP}}^{4}<\alpha k_{7}$. For $\alpha=\frac{3}{5}$ the function $L_{2}$ coincides with $L_{1}$. Another interesting choice is $\alpha=\frac{1}{2}$. In that case $L_{2}$ is monotone decreasing on the region defined by the inequality $k_{4} x_{\mathrm{GAP}}^{4}<k_{7}$. It follows that if a solution initially satisfies

$$
x_{\mathrm{RuBP}}+\frac{1}{2}\left(x_{\mathrm{PGA}}+x_{\mathrm{DPGA}}+x_{\mathrm{GAP}}\right)+x_{\mathrm{Ru} 5 \mathrm{P}}<\frac{1}{2}\left(\frac{k_{7}}{k_{4}}\right)^{\frac{1}{4}}
$$

then it tends to zero as $t \rightarrow \infty$.

It has now been shown that in the case $k_{2}>5 k_{6}$ there is an open set of initial data for which the corresponding solutions tend to the origin as $t \rightarrow \infty$. The argument just given also provides some information about the basin of attraction of the origin, which is more than could be concluded from the fact that the origin is a hyperbolic sink. There is also an open set of initial data for which all concentrations $x_{i}$ tend to infinity as $t \rightarrow \infty$. The proof of this statement is given in Section 3 .

In 22] stoichiometric coefficients are considered which are slightly different from those in [9]. While 22] uses Michaelis-Menten kinetics it is possible to take mass action kinetics with the stoichiometric coefficients of [22]. This leads to a system which is called MAZ in what follows. It differs from the system MA only by the facts that the terms $-5 k_{4} x_{\mathrm{GAP}}^{5}$ and $3 k_{4} x_{\mathrm{GAP}}^{5}$ are replaced by $-k_{4} x_{\mathrm{GAP}}$ and $\frac{3}{5} k_{4} x_{\mathrm{GAP}}$ respectively. In terms of the reaction network, the system MA arises from the system MAZ by multiplying the stoichiometric coefficients in one of the reactions by a constant factor so that they become integers. For the system MAZ the set $\bar{S}$ is positively invariant. The right hand side of the system is $C^{1}$ due to the fact that all the stoichiometric coefficients on the left hand sides of reactions are integers. The arguments used to prove that solutions starting in $\bar{S}$ have no $\omega$-limit points on the boundary of $S$ other than the origin for the system MA generalize easily to give the same statements for the system MAZ. Since the latter system is linear all solutions exist globally. The function $L_{1}$ of (2.11) is a Lyapunov function for the system MAZ when $k_{2} \leq 5 k_{6}$ since it also satisfies the equation (2.12) in this case. It follows that all the statements about the system MA derived using $L_{1}$ also hold for the system MAZ. In the latter case

$$
\frac{d L_{2}}{d t}=-\frac{1}{2}\left[k_{6}-(2 \alpha-1) k_{2}\right] x_{\mathrm{PGA}}-\left[\alpha k_{7}-\left(\frac{3}{5}-\alpha\right) k_{4}\right] x_{\mathrm{GAP}}
$$

Thus if $(2 \alpha-1) k_{2} \geq k_{6}$ and $(3-5 \alpha) k_{4} \geq 5 \alpha k_{7}$ and at least one of these two inequalities is strict then $-L_{2}$ is a Lyapunov function. The equations for stationary solutions may be analysed in this case in a similar way to what was done for the system MA. Four of the relations obtained are $x_{\mathrm{Ru} 5 \mathrm{P}}=\frac{k_{1}}{k_{5}} x_{\mathrm{RuBP}}$, 
$x_{\mathrm{PGA}}=\frac{2 k_{1}}{k_{2}+k_{6}} x_{\mathrm{RuBP}}, x_{\mathrm{DPGA}}=\frac{2 k_{1} k_{2}}{k_{3}\left(k_{2}+k_{6}\right)} x_{\mathrm{RuBP}}, x_{\mathrm{GAP}}=\frac{5 k_{1}}{3 k_{4}} x_{\mathrm{RuBP}}$. Substituting these relations in the remaining equation gives:

$$
5\left(k_{2}+k_{6}\right)\left(k_{4}+k_{7}\right)=6 k_{2} k_{4} \text {. }
$$

Thus there are stationary solutions only when this relation is satisfied and when it is satisfied there is a whole one-dimensional subspace of them. This situation is not surprising since the equations are linear in this case. It is natural to examine the eigenvalues of the matrix on the right hand side of the equation. The characteristic polynomial is

$$
\left(k_{1}+\lambda\right)\left(k_{3}+\lambda\right)\left(k_{5}+\lambda\right)\left(k_{2}+k_{6}+\lambda\right)\left(k_{4}+k_{7}+\lambda\right)-\frac{6}{5} k_{1} k_{2} k_{3} k_{4} k_{5} .
$$

As in the case of the system MA all the coefficients in this polynomial are positive except possibly for the constant term, which is

$$
k_{1} k_{3} k_{5}\left[\left(k_{2}+k_{6}\right)\left(k_{4}+k_{7}\right)-\frac{6}{5} k_{2} k_{4}\right] \text {. }
$$

When the expression in brackets is negative there is a positive real eigenvalue and there exists at least one solution which tends to infinity as $t \rightarrow \infty$ since the origin has a non-trivial linear unstable manifold. If

$$
\left(k_{2}+k_{6}\right)\left(k_{4}+k_{7}\right)<\frac{6}{5} k_{2} k_{4}
$$

then $\alpha$ can be chosen so that $-L_{2}$ is a Lyapunov function. Hence in that case the origin does not belong to the $\omega$-limit point of any solution. Since other $\omega$-limit points on the boundary of the positive orthant have already been ruled out it follows that all solutions tend to infinity. The results for the systems MA and MAZ are now collected as a theorem.

Theorem 1 If $k_{2} \leq 5 k_{6}$ then all solutions of MA and MAZ tend to the origin as $t \rightarrow \infty$. If $k_{2}>5 k_{6}$ there is a non-empty open set of initial data for MA for which the corresponding solutions tend to the origin as $t \rightarrow \infty$ and a non-empty open set of initial data for which the corresponding solutions tend to infinity as $t \rightarrow \infty$. If $k_{2}>5 k_{6}$ then there is at least one solution of MAZ which tends to infinity as $t \rightarrow \infty$. If $k_{4}\left(k_{2}-5 k_{6}\right)>5\left(k_{2}+k_{6}\right) k_{7}$ then all solutions of MAZ tend to infinity as $t \rightarrow \infty$.

\section{Solutions which tend to infinity}

In this section it is shown that if $k_{2}>5 k_{6}$ there exist solutions of (2.1)-(2.5) for which all $x_{i}$ tend to infinity as $t \rightarrow \infty$. In fact there is a non-empty open set of initial data for which the corresponding solutions behave in this way.

Theorem 2 If $k_{2}>5 k_{6}$ there is a non-empty open set of initial data for the system MA for which the corresponding solutions tend to infinity as $t \rightarrow \infty$ and 
have the asymptotics

$$
\begin{aligned}
& x_{1}(t)=A e^{\alpha t}+\ldots \\
& x_{2}(t)=A\left(V_{2} / V_{1}\right) e^{\alpha t}+\ldots \\
& x_{3}(t)=A\left(V_{3} / V_{1}\right) e^{\alpha t}+\ldots \\
& x_{4}(t)=\left[A\left(V_{3} / V_{1}\right) \xi_{s}\right]^{\frac{1}{5}} e^{\frac{\alpha t}{5}}+\ldots \\
& x_{5}(t)=A\left(V_{5} / V_{1}\right) e^{\alpha t}+\ldots
\end{aligned}
$$

Here $\alpha, \xi_{s}$ and the $V_{i}$ are fixed positive constants and $A$ is a constant depending on the solution.

Proof For the proof it is useful to introduce the quantity $\xi=\frac{x_{4}^{5}}{x_{3}}$. Its evolution equation is given by

$$
\frac{d \xi}{d t}=5\left(x_{3} \xi\right)^{\frac{4}{5}}\left[k_{3}-5 k_{4} \xi+\xi^{\frac{1}{5}} x_{3}^{-\frac{4}{5}}\left(\frac{1}{5} k_{3}-k_{7}-\frac{k_{2} x_{2}}{5 x_{3}}\right)\right] .
$$

Let $\bar{x}$ be the vector with components $x_{i}$ for $i=1,2,3,5$. Then four of the evolution equations can be rewritten as

$$
\frac{d \bar{x}}{d t}=M \bar{x}+R
$$

Here the only non-zero component of the vector $R$ is the last one and it is equal to $3 k_{4} x_{3}\left(\xi-\xi_{s}\right), \xi_{s}=\frac{k_{3}}{5 k_{4}}$ and

$$
M=\left[\begin{array}{cccc}
-k_{1} & 0 & 0 & k_{5} \\
2 k_{1} & -\left(k_{2}+k_{6}\right) & 0 & 0 \\
0 & k_{2} & -k_{3} & 0 \\
0 & 0 & \frac{3}{5} k_{3} & -k_{5}
\end{array}\right] .
$$

Equations (3.6) and (3.7) are equivalent to the original system. The solutions to be constructed are obtained as fixed points of a mapping depending on parameters. To define this mapping some manipulations of the basic equations (3.6) and (3.7) are necessary. The equation (3.6) can be rewritten as

$$
\frac{d \xi}{d t}=25 k_{4}\left(x_{3} \xi\right)^{\frac{4}{5}}\left(\xi_{s}-\xi\right)+5 \xi\left(\frac{1}{5} k_{3}-k_{7}-\frac{k_{2} x_{2}}{5 x_{3}}\right) .
$$

The first term on the right hand side can be split into a leading term and a remainder with the result that the whole right hand side can be written as

$$
25 k_{4}\left(x_{3} \xi_{s}\right)^{\frac{4}{5}}\left(\xi_{s}-\xi\right)+F(\bar{x}, \xi)
$$

where

$$
F(\bar{x}, \xi)=25 k_{4}\left(\bar{x}_{3}\right)^{\frac{4}{5}}\left[\xi^{\frac{4}{5}}-\xi_{s}^{\frac{4}{5}}\right]\left(\xi_{s}-\xi\right)+5 \xi\left(\frac{1}{5} k_{3}-k_{7}-\frac{k_{2} \bar{x}_{2}}{5 \bar{x}_{3}}\right) .
$$


The equation (3.7) can be solved by variation of constants to give

$$
\bar{x}(t)=\exp (M t) \bar{x}(0)+\int_{0}^{t} \exp (M(t-s)) R[\bar{x}, \xi](s) d s .
$$

The function $R[\bar{x}, \xi](s)$ depends on the functions $\bar{x}(s)$ and $\xi(s)$.

The matrix $M$ is of the type considered in Appendix 2. Its determinant is $k_{1} k_{3} k_{5}\left(k_{6}-\frac{1}{5} k_{2}\right)$. Thus when $k_{2}>5 k_{6}$ it has exactly one positive eigenvalue $\alpha$ and all its other eigenvalues have real parts less than $\alpha$. Moreover, there is an eigenvector $V$ with eigenvalue $\alpha$ all of whose components are positive. Thus

$$
\exp (M t) \bar{x}(0)=\mu_{1} e^{\alpha t} V+\psi\left(t ; \mu_{2}, \mu_{3}, \mu_{4}\right)
$$

where the components of the function $\psi$ are of the form

$$
\psi_{i}\left(t ; \mu_{2}, \mu_{3}, \mu_{4}\right)=\sum_{j=2}^{4} \mu_{j} W_{i j} e^{\beta_{j} t} \sum_{k \geq 0} \nu_{j k} t^{k} .
$$

Here the $\beta_{j}$ are the eigenvalues of $M$ other than $\alpha$, the $W_{i j}$ are the components of the eigenvectors other than $V$ and the $\nu_{j k}$ are constants which are only non-zero for $k>0$ if the eigenvalue $\beta_{j}$ has multiplicity greater than one. The function $\psi$ depends linearly on the parameters $\mu_{2}, \mu_{3}$ and $\mu_{4}$ and satisfies $\psi\left(t ; \mu_{2}, \mu_{3}, \mu_{4}\right)=$ $O\left(e^{(\alpha-\epsilon) t}\right)$ as $t \rightarrow \infty$ for some $\epsilon>0$.

From the formula for $\bar{x}$ we get

$$
x_{3}(t)=\mu_{1} e^{\alpha t} V_{3}+e_{3} \cdot \psi\left(t ; \mu_{2}, \mu_{3}, \mu_{4}\right)+\int_{0}^{t} e_{3} \cdot \exp (M(t-s)) R[\bar{x}, \xi](s) d s .
$$

This can be used to split the quantity $x_{3}$ in the first term of (3.10) into a part containing the leading term in (3.15) and a remainder term. The result is

$$
\frac{d \xi}{d t}=25 k_{4}\left(\mu_{1} V_{3} \xi_{s} e^{\alpha t}\right)^{\frac{4}{5}}\left(\xi_{s}-\xi\right)+G[\bar{x}, \xi, t]
$$

where

$$
G[\bar{x}, \xi, t]=25 k_{4} \xi_{s}^{\frac{4}{5}}\left[\bar{x}_{3}^{\frac{4}{5}}-\left(\mu_{1} V_{3} e^{\alpha t}\right)^{\frac{4}{5}}\right]\left(\xi_{s}-\xi\right)+F(\bar{x}, \xi) .
$$

The idea is that $G$ contains all the contributions to (3.6) which can be considered small.

Equation (3.16) can also be treated by variation of constants. Let

$$
\Phi(t)=\exp \left(-\frac{125 k_{4}}{4 \alpha}\left[\left(\mu_{1} V_{3} \xi_{s} e^{\alpha t}\right)^{\frac{4}{5}}-1\right]\right) .
$$

Then

$$
\xi(t)=\xi_{s}+\eta_{0} \Phi(t)+\int_{0}^{t} \frac{\Phi(t)}{\Phi(s)} G[\bar{x}, \xi, s] d s
$$


with an arbitrary constant $\eta_{0}$. Finally (3.12) can be rewritten as

$$
\bar{x}(t)=\mu_{1} e^{\alpha t} V+\psi\left(t ; \mu_{2}, \mu_{3}, \mu_{4}\right)+\int_{0}^{t} \exp (M(t-s)) R[\bar{x}, \xi](s) d s .
$$

The integral equations (3.19) and (3.20) are those which are used for the fixed point argument.

Let $X$ be the set of continuous functions $(\bar{x}(t), \xi(t))$ defined on the interval $[0, \infty)$ which satisfy the inequalities

$$
\frac{\left|\bar{x}(t)-\mu_{1} e^{\alpha t} V\right|}{e^{\alpha t}} \leq \delta \mu_{1}, \quad\left|\xi(t)-\xi_{s}\right| e^{\frac{4}{5} \alpha t} \leq K
$$

for positive constants $\mu_{1}, \delta$ and $K$ which are restricted by some additional conditions in the fixed point argument. Denote the right hand sides of equations (3.19) and (3.20) by $\mathcal{T}_{1}(\xi, \bar{x})$ and $\mathcal{T}_{2}(\xi, \bar{x})$, respectively and let $\mathcal{T}=\left(\mathcal{T}_{1}, \mathcal{T}_{2}\right)$. Then $\xi$ and $\bar{x}$ solve (3.19) and (3.20) if and only if $(\xi, \bar{x})$ is a fixed point of $\mathcal{T}$. In order to ensure that the mapping $\mathcal{T}$ is well-defined on $X$ it suffices to assume that $\delta \leq \frac{1}{2} \min _{i} V_{i}$ and that $K \leq \frac{1}{2} \xi_{s}$ since these inequalities imply the positivity of $\bar{x}$ and $\xi$. The aim is to show that for a suitable choice of the constants $\mu_{1}$, $\mu_{2}, \mu_{3}, \mu_{4}, \eta_{0}, \delta$ and $K$ this rule defines a mapping $\mathcal{T}$ from $X$ to itself.

For convenience let $\mathcal{T}(\xi, \bar{x})=(\zeta, y)$. The quantities $\zeta$ and $y$ should now be estimated under the assumptions (3.21). The quantity $\zeta-\xi_{s}$ is estimated first. It can be written as a sum $\sum_{i=1}^{5} Q_{i}$ where the individual terms are defined as follows. $Q_{1}$ denotes the second term on the right hand side of (3.19). $Q_{2}$ denotes the contribution to the right hand side of (3.19) coming from the first term on the right hand side of (3.17). The contribution to the right hand side of (3.19) coming from the second term on the right hand side of (3.17) is $Q_{3}+Q_{4}+Q_{5}$. The three summands in this last expression come from three summands in $F$. The expression $Q_{3}$ is the contribution coming from the first term in (3.11). $Q_{4}$ is the contribution from the first two terms in the bracket on the right hand side of (3.11) and $Q_{5}$ is the contribution from the third term in that bracket. As a first step towards estimating $\zeta-\xi_{s}$ consider the following identity which holds for any positive constants $A$ and $\gamma$.

$$
\frac{d}{d t}\left(\gamma^{-1} A^{-1} e^{-\gamma t} \exp \left(A e^{\gamma t}\right)\right)=\exp \left(A e^{\gamma t}\right)\left(1-A^{-1} e^{-\gamma t}\right) .
$$

If $A \geq 2$ then the second factor on the right hand side of this equation is no smaller than one half for any $t \geq 0$. Hence integrating this relation from 0 to $t$ gives

$$
\int_{0}^{t} \exp \left(A e^{\gamma s}\right) d s \leq 2 \gamma^{-1} A^{-1} e^{-\gamma t} \exp \left(A e^{\gamma t}\right) .
$$

Thus

$$
\begin{aligned}
& \left|\zeta-\xi_{s}\right| \leq \Phi(t)\left[\left|\eta_{0}\right|+\|G\|_{L^{\infty}} \int_{0}^{t} \frac{1}{\Phi(s)} d s\right] \\
& \leq \frac{2}{25 k_{4}}\left(\mu_{1} V_{3} \xi_{s}\right)^{-\frac{4}{5}}\|G\|_{L^{\infty}} e^{-\frac{4 \alpha}{5} t}+\left|\eta_{0}\right| \Phi(t) .
\end{aligned}
$$


The integral has been estimated using (3.23) with $A=\left(\frac{125 k_{4}}{4 \alpha}\right)\left(\mu_{1} V_{3} \xi_{s}\right)^{\frac{4}{5}}$ and $\gamma=\frac{4 \alpha}{5}$. Assuming $\mu_{1}$ sufficiently large ensures that the lower bound on $A$ required for (3.23) to hold is satisfied. Next $G$ is estimated.

$$
\|G\|_{L^{\infty}} \leq 20 k_{4} \xi_{s}^{\frac{4}{5}} K\left(\mu_{1} V_{3}\right)^{\frac{4}{5}} V_{3}^{-1} \sup \left\{1,\left(V_{3}-\delta\right)^{-\frac{2}{5}}\right\} \delta+\|F\|_{L^{\infty}}
$$

Choosing $\delta$ small enough allows $Q_{2}$ to be bounded by $\frac{K}{5} e^{-\frac{4}{5} \alpha t}$. The first term in the expression for $F$ can be bounded by

$$
C k_{4} \mu_{1}^{\frac{4}{5}} e^{\frac{4}{5} \alpha t}\left(V_{3}+\delta\right)^{\frac{4}{5}}\left(\xi-\xi_{s}\right)^{2} \leq C k_{4} \mu_{1}^{\frac{4}{5}} K^{2}\left(V_{3}+\delta\right)^{\frac{4}{5}} e^{-\frac{4 \alpha}{5} t}
$$

for a numerical constant $C$. Choosing $K$ sufficiently small allows $Q_{3}$ to be bounded by $\frac{K}{5} e^{-\frac{4}{5} \alpha t}$. In the second term in $F$ the first two contributions can be bounded by $2 \xi_{s}\left(k_{3}+5 k_{7}\right)$. Choose $\mu_{1}$ large enough so that

$$
\frac{4}{5 k_{4}}\left(\mu_{1} V_{3} \xi_{s}\right)^{-\frac{4}{5}} \xi_{s}\left(k_{3}+5 k_{7}\right) \leq K
$$

Then it follows that $Q_{4}$ is no greater than $\frac{K}{5} e^{-\frac{4}{5} \alpha t}$. The third contribution to $F$ can be bounded by $2 \xi_{s} k_{2}\left|\frac{x_{2}}{x_{3}}\right|$. Now

$$
\left|\frac{x_{2}}{x_{3}}\right| \leq \frac{V_{2}+\delta e^{-2 \alpha t}}{V_{3}-\delta e^{-2 \alpha t}} .
$$

To get a lower bound for the denominator in this expression it is assumed that $\delta \leq \frac{1}{2} V_{3}$. Then

$$
\left|\frac{x_{2}}{x_{3}}\right| \leq \frac{2 V_{2}+V_{3}}{V_{3}} .
$$

If $\mu_{1}$ is chosen large enough then $Q_{5}$ is bounded by $\frac{K}{5} e^{-\frac{4}{5} \alpha t}$. To control the quantity $Q_{1}$ assume that $\mu_{1}$ is so large that

$$
\frac{125 k_{4}}{4 \alpha}\left[\left(\mu_{1} V_{3} \xi_{s}\right)^{\frac{4}{5}} e^{\frac{4 \alpha}{5} t}-1\right] \geq \frac{4 \alpha}{5} t .
$$

for all $t \geq 0$. Then $\left|\eta_{0}\right| \Phi(t) \leq\left|\eta_{0}\right| e^{-\frac{4 \alpha}{5} t}$. Choose $\eta_{0}$ to be no larger in modulus than $\frac{K}{5}$. Then combining the estimates shows that for $K$ and $\delta$ sufficiently small and $\mu_{1}$ sufficiently large the second defining inequality of the set $X$ is satisfied by $\zeta$.

Next $y-\mu_{1} e^{\alpha t} V$ is estimated. The function $\psi$ can be bounded by an expression of the form $C e^{(\alpha-\epsilon) t}$ where the constant $C$ depends only on the matrix $M$ and $\epsilon$ is a positive constant. Since $\psi$ is linear in the parameters $\mu_{2}, \mu_{3}$ and $\mu_{4}$ the constant $C$ can be made as small as desired by making these parameters small. Thus it can be ensured that $e^{-\alpha t}|\psi(t)| \leq \frac{\mu_{1} \delta}{2}$. The integral term in (3.20) can be estimated by an expression of the form $C \mu_{1} K e^{\alpha t}$. This can be made as small as desired compared to $\delta \mu_{1} e^{\alpha t}$ by choosing $K$ small. Thus the first defining inequality of the set $X$ is satisfied by $y$. 
It follows that for a suitable choice of the parameters $\mathcal{T}$ maps $X$ into itself. Let $\bar{x}^{0}(t)=\mu_{1} e^{\alpha t} V$ and $\xi^{0}(t)=\xi_{s}$. Define a sequence $\left(\xi^{n}, \bar{x}^{n}\right)$ recursively by $\left(\xi^{n+1}, \bar{x}^{n+1}\right)=\mathcal{T}\left(\left(\xi^{n}, \bar{x}^{n}\right)\right)$. The sequences $\bar{x}^{n}$ and $\xi^{n}$ are uniformly bounded on compact subsets. It then follows from the definition of $\mathcal{T}$ that their time derivatives are uniformly bounded on compact subsets. By the Arzela-Ascoli theorem there is a subsequence $\left(\xi^{n_{r}}, \bar{x}^{n_{r}}\right)$ which converges uniformly to a limit on compact subsets. It is possible to pass to the limit in the integral equations defining the iteration to see that the limit is a fixed point of $\mathcal{T}$ and hence the desired solution. The solution is uniquely determined by the parameters $\mu_{i}$ and $\eta_{0}$. The mapping from these parameters to the initial data for the solution at $t=0$ is a diffeomorphism onto its image. Thus the set of solutions constructed in this way corresponds to an open set of initial data. This completes the proof of the theorem.

\section{Michaelis-Menten via mass action kinetics}

Next the system (10) of 9 is considered. For the convenience of the reader we reproduce the essential equations here:

$$
\begin{aligned}
& \dot{x}_{\mathrm{RuBP}}=k_{15} x_{\mathrm{Ru}_{5 \mathrm{PE}_{5}}}-k_{1} x_{\mathrm{RuBP}} x_{\mathrm{E}_{1}}+k_{2} x_{\mathrm{RuBPE}_{1}}, \\
& \dot{x}_{\mathrm{RuBPE}_{1}}=k_{1} x_{\mathrm{RuBP}} x_{\mathrm{E}_{1}}-\left(k_{2}+k_{3}\right) x_{\mathrm{RuBPE}_{1}}, \\
& \dot{x}_{\mathrm{PGA}}=2 k_{3} x_{\mathrm{RuBPE}_{1}}-k_{4} x_{\mathrm{PGA}} x_{\mathrm{E}_{2}}+k_{5} x_{\mathrm{PGAE}_{2}} \\
& -k_{16} x_{\mathrm{PGA}} x_{\mathrm{E}_{6}}+k_{17} x_{\mathrm{PGAE}_{6}} \text {, } \\
& \dot{x}_{\mathrm{PGAE}_{2}}=k_{4} x_{\mathrm{PGA}} x_{\mathrm{E}_{2}}-\left(k_{5}+k_{6}\right) x_{\mathrm{PGAE}_{2}} \text {, }
\end{aligned}
$$

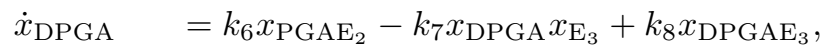

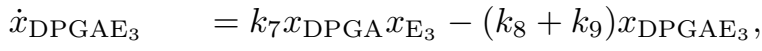

$$
\begin{aligned}
& \dot{x}_{\mathrm{GAP}}=k_{9} x_{\mathrm{DPGAE}_{3}}-5 k_{10} x_{\mathrm{GAP}}^{5} x_{\mathrm{E}_{4}}+5 k_{11} x_{\mathrm{GAPE}_{4}} \\
& -k_{19} x_{\mathrm{GAP}} x_{\mathrm{E}_{7}}+k_{20} x_{\mathrm{GAPE}_{7}} \text {, } \\
& \dot{x}_{\mathrm{GAPE}_{4}}=k_{10} x_{\mathrm{GAP}}^{5} x_{\mathrm{E}_{4}}-\left(k_{11}+k_{12}\right) x_{\mathrm{GAPE}_{4}} \text {, } \\
& \dot{x}_{\mathrm{Ru} 5 \mathrm{P}}=-k_{13} x_{\mathrm{Ru} 5 \mathrm{P}} x_{\mathrm{E}_{5}}+k_{14} x_{\mathrm{Ru}_{5 \mathrm{PE}_{5}}}+3 k_{12} x_{\mathrm{GAPE}_{4}}, \\
& \dot{x}_{\mathrm{Ru}_{5 \mathrm{PE}_{5}}}=k_{13} x_{\mathrm{Ru} 5 \mathrm{P}} x_{\mathrm{E}_{5}}-\left(k_{14}+k_{15}\right) x_{\mathrm{Ru}_{5 \mathrm{PE}_{5}}} \text {, } \\
& \dot{x}_{\mathrm{PGAE}_{6}}=k_{16} x_{\mathrm{PGA} x_{\mathrm{E}_{6}}}-\left(k_{17}+k_{18}\right) x_{\mathrm{PGAE}_{6}} \text {, } \\
& \dot{x}_{\mathrm{GAPE}_{7}}=k_{19} x_{\mathrm{GAP}} x_{\mathrm{E}_{7}}-\left(k_{20}+k_{21}\right) x_{\mathrm{GAPE}_{7}} .
\end{aligned}
$$

The equations for the concentrations of the free enzymes have been omitted since they can easily be reconstructed. As explained below, this could be made into a closed system by using the conservation of the total quantity of each enzyme. This has not been done so as to prevent the equations becoming even longer. The kinetics is called Michaelis-Menten represented in terms of mass action in [9] and is called the system MM-MA here. The unknowns are of three types. There are the concentrations of free substrates, which are denoted by the same variables as in the system MA. There are the concentrations $x_{\mathrm{E}_{\alpha}}$ of 
the seven enzymes corresponding to the seven reactions in the system. Finally, there are the concentrations of the complexes formed when the enzymes bind to their substrates. The complex formed when the substrate $A_{i}$ binds to the enzyme $E_{\alpha}$ is denoted in what follows by $A_{i} E_{\alpha}$. Since in some reactions $r(\alpha)>1$ molecules of the substrate bind to the enzyme this might be denoted instead by $A_{i}^{r(\alpha)} E_{\alpha}$. Since, however, the exponent $r(\alpha)$ is uniquely determined by $\alpha$ we chose the shorter notation to prevent certain formulae becoming even more cumbersome than they already are. It should be warned that the word 'complex' is used in two different ways in the literature on reaction networks. The first meaning is the one just introduced. The other is a formal linear combination of the chemical species which is on the left or right hand side of a reaction. To distinguish these two concepts in what follows they will be referred to as 'enzyme-substrate complex' and 'reaction complex' respectively.

The total concentration of each enzyme (free plus bound) is a conserved quantity and is denoted by $\rho_{\alpha}$. It follows as for the system MA that the set $\bar{S}$ is invariant. The question, whether solutions starting in $S$ can have $\omega$-limit points on the boundary is a little more complicated than for the system MA. Note first that there is a five-dimensional set $A_{1}$ of stationary solutions in $\bar{S}$ defined by setting the concentrations of all enzymes to zero together with those of the corresponding complexes. This is the set where all $\rho_{\alpha}$ are zero. This set cannot contain any $\omega$-limit point of a solution with positive initial data, since for a solution of that type the $\rho_{\alpha}$ are positive. The conservation of the $\rho_{\alpha}$ defines invariant affine subspaces of $S$ of codimension seven. It is elementary to show that the stoichiometric matrix has rank twelve so that these subspaces are the stoichiometric compatibility classes. Call one of these subspaces $S_{E}$. Another set of stationary solutions $A_{2}$, of dimension seven, is defined by setting the concentration of all substrates and all enzyme-substrate complexes to zero. Any subspace $S_{E}$ intersects this set of stationary solutions in precisely one point. Consider a solution $x(t)$ which is positive and which has an $\omega$-limit point $x^{*}$ on the boundary of $S$. The solution $y(t)$ passing through $x^{*}$ lies entirely in the boundary of $S$. If the concentration of any free enzyme $E_{\alpha}$ vanishes at $x^{*}$ then, by the conservation laws, the concentration of the corresponding substrate-enzyme complex is non-zero. It follows that the time derivative of the concentration of $E_{\alpha}$ is positive, a contradiction. Thus it can be concluded that the concentrations of all free enzymes are non-zero at $x^{*}$. Suppose that $x^{*} \notin A_{2}$. If all substrates had zero concentration at $x^{*}$ then at least one enzymesubstrate complex would have to be non-zero and the evolution equation for that substrate would give a contradiction. Thus at least one substrate must have nonzero concentration. Then the evolution equation for a complex of that substrate with any enzyme implies that the concentration of that complex must be nonzero. Since $x^{*}$ is on the boundary of $S$ it is not possible that the concentrations of all substrates are non-zero. From this point on it is possible to argue as in the corresponding proof for the system MA to obtain a contradiction. It can be concluded that any $\omega$-limit point of a solution starting in $S$ must either be a point of $S$ or belong to $A_{2}$. The conservation laws show that the $\omega$-limit set 
contains at most one point of $A_{2}$.

The system (4.1)-(4.12) has the property that all the variables representing the concentrations of enzymes or enzyme-substrate complexes are bounded due to the conservation laws for the quantities $\rho_{\alpha}$. On the other hand, the quantities on the right hand sides of the evolution equations for all other variables are all either non-positive or linear in the quantities which are not known to be bounded. It follows from this that all solutions exist globally in the future. Summing up:

Proposition 2 A solution of the system (4.1)-(4.12) (the system MM-MA) with positive initial data exists globally to the future, remains positive and has no $\omega$-limit points on the boundary of $S$ except possibly a single point of the set $A_{2}$. The conclusions listed for the system MM-MA in this proposition also hold for the analogous system MM-MAZ defined using the stoichiometric coefficients of 22] and can be proved in the same way.

For $i=1,2,3,4,5$ let $\tilde{x}_{i}$ be the sum of the concentration of the free substrate $i$ and its concentrations within its complexes with enzymes. Note that here it is necessary to take into account that in general the complex contains several molecules of the substrate. Then $A_{2}$ is the subset of $\bar{S}$ where all $\bar{x}_{i}$ vanish. These quantities satisfy the evolution equations

$$
\begin{aligned}
\frac{d \tilde{x}_{1}}{d t} & =k_{15} x_{\mathrm{Ru} \mathrm{PE}_{5}}-k_{3} x_{\mathrm{RuBPE}_{1}}, \\
\frac{d \tilde{x}_{2}}{d t} & =2 k_{3} x_{\mathrm{RuBPE}_{1}}-k_{6} x_{\mathrm{PGAE}_{2}}-k_{18} x_{\mathrm{PGAE}_{6}}, \\
\frac{d \tilde{x}_{3}}{d t} & =k_{6} x_{\mathrm{PGAE}_{2}}-k_{9} x_{\mathrm{DPGAE}_{3}}, \\
\frac{d \tilde{x}_{4}}{d t} & =k_{9} x_{\mathrm{DPGAE}_{3}}-5 k_{12} x_{\mathrm{GAPE}_{4}}-k_{21} x_{\mathrm{GAPE}_{7}}, \\
\frac{d \tilde{x}_{5}}{d t} & =3 k_{12} x_{\mathrm{GAPE}_{4}}-k_{15} x_{\mathrm{Ru}_{\mathrm{PE}}} .
\end{aligned}
$$

Let $\tilde{L}_{1}$ be the quantity obtained by replacing $x_{i}$ by $\tilde{x}_{i}$ in the expression for the function $L_{1}$ introduced for the system MA. Then

$$
\frac{d \tilde{L}_{1}}{d t}=-\frac{1}{2}\left(k_{18} x_{\mathrm{PGAE}_{6}}-\frac{1}{5} k_{6} x_{\mathrm{PGAE}_{2}}\right)-\frac{3}{5} k_{21} x_{\mathrm{GAPE}_{7}} .
$$

This shows that $\tilde{L}_{1}$ is a Lyapunov function on the region where the quantity in brackets in (4.18) is non-negative. It will now be shown that this can be used to prove that certain solutions tend to zero as $t \rightarrow \infty$.

Proposition 3 A solution of the system MM-MA (the system (4.1)-(4.12) with $k_{17}+k_{18}<k_{5}+k_{6}$ which satisfies the inequalities (4.19) and $k_{4} k_{6} \rho_{2}<$ $k_{16} k_{18}\left(\rho_{6}-2 \bar{L}_{1}(0)\right)$ converges to a point of $A_{2}$ as $t \rightarrow \infty$.

Proof For a solution satisfying the assumptions of the proposition the quantity in brackets in (4.18) is initially positive, i.e.

$$
k_{6} x_{\mathrm{PGAE}_{2}}(0)<5 k_{18} x_{\mathrm{PGAE}_{6}}(0) .
$$


The evolution equations for the concentrations occurring in this inequality are

$$
\begin{aligned}
& \frac{d x_{\mathrm{PGAE}_{2}}}{d t}=k_{4} x_{\mathrm{PGA}_{\mathrm{E}}} x_{\mathrm{E}_{2}}-\left(k_{5}+k_{6}\right) x_{\mathrm{PGAE}_{2}}, \\
& \frac{d x_{\mathrm{PGAE}_{6}}}{d t}=k_{16} x_{\mathrm{PGA}_{\mathrm{A}}} x_{\mathrm{E}_{6}}-\left(k_{17}+k_{18}\right) x_{\mathrm{PGAE}_{6}} .
\end{aligned}
$$

Let $t_{*}$ be supremum of times for which $k_{6} x_{\mathrm{PGAE}_{2}}(t)<5 k_{18} x_{\mathrm{PGAE}_{6}}(t)$ holds on the interval $\left[0, t_{*}\right)$. Using the fact that $k_{17}+k_{18}<k_{5}+k_{6}$ the sum of the contributions of the second terms on the right hand sides of the evolution equations for $x_{\mathrm{PGAE}_{2}}$ and $x_{\mathrm{PGAE}_{6}}$ to the evolution equation for $5 k_{18} x_{\mathrm{PGAE}_{6}}-$ $k_{6} x_{\mathrm{PGAE}_{2}}$ is positive when $t=t_{*}$. Now $k_{4} x_{\mathrm{PGA}} x_{\mathrm{E}_{2}} \leq k_{4} x_{\mathrm{PGA}} \rho_{2}$ and

$$
k_{16} x_{\mathrm{PGA}} x_{\mathrm{E}_{6}}=k_{16} x_{\mathrm{PGA}}\left(\rho_{6}-x_{\mathrm{PGAE}_{6}}\right) \geq k_{16} x_{\mathrm{PGA}}\left(\rho_{6}-2 \tilde{L}_{1}(0)\right) .
$$

Thus due to the inequality $k_{4} k_{6} \rho_{2}<k_{16} k_{18}\left(\rho_{6}-2 \tilde{L}_{1}(0)\right)$ the assumption that $t_{*}$ is finite leads to a contradiction. In addition it can be seen that in this case any $\omega$-limit point of the solution must satisfy $x_{\mathrm{PGA}}=0$ and hence belong to $A_{2}$. This gives the conclusion of the proposition.

Next it will be shown that the system MM-MA has solutions for which the concentrations of the substrates tend to infinity at late times. To do this it is most economical to do the calculations in the framework of a class of reaction networks wider than those describing the Calvin cycle. Consider a system of chemical reactions as defined by sets of species, reaction complexes and reactions. This will be called the basic reaction network. It is possible to build a new network by replacing each reaction in the basic network by a Michaelis-Menten scheme containing a substrate (the species from the basic network), an enzyme and a substrate-enzyme complex. Applying mass action kinetics to the extended network gives 'Michaelis-Menten expressed in terms of mass action' kinetics or, for short, MM-MA kinetics. In this way starting from the basic network we get a system of ordinary differential equations called the MM-MA system. It contains reaction constants for each reaction in the extended system as parameters. Call the substrates $A_{i}$ and the enzymes $E_{\alpha}$ for some indices $i$ and $\alpha$. The complex formed when these bind to each other is denoted by $A_{i} E_{\alpha}$.

Some restrictions will now be made on the basic set of chemical reactions.

1. Each reaction complex in the basic network contains only one species

2. The set of substrates and the set of enzymes are disjoint. This rules out the MAP kinase cascade [12.

3. Each enzyme catalyses only one reaction. This rules out systems with enzyme sharing such as the multiple futile cycle [20].

When there are $n$ species and $r$ reactions in the basic network then the number of species in the corresponding MM-MA system is $n+2 r$. There are $n$ substrates, $r$ free enzymes and $r$ substrate-enzyme complexes. There are $3 r$ reactions. 
In the motivating example for this work the basic system is defined by the equations (2.1)-(2.5) describing the Calvin cycle. It has five species. There are seven reactions and so there are nineteen species in the corresponding MM-MA system. When a system is so big it is not very efficient to write it out explicitly when analysing it. It can be more useful to treat it as an example of a class of systems characterized by some particular structural properties. This is the motivation for considering a more general class of systems here. Note that the first restriction above rules out the more detailed models of the Calvin cycle given in [17] and [18]. It also rules out the homogeneous case of the model with diffusion considered in $[9$. It will be seen in Section 6 that in fact all solutions of the latter system are bounded.

The main theme of what follows is solutions of an MM-MA system in which the concentrations of all substrates tend to infinity as $t \rightarrow \infty$. In fact they all tend to infinity linearly in time. In the solutions of interest here the concentration of each free enzyme tends to zero as $t \rightarrow \infty$ and almost all the enzyme becomes bound to the substrate at late times. A class of networks are considered which are called autocatalytic. It is shown that for MM-MA systems arising from networks satisfying this additional property, which is defined later, there are large classes of solutions of the type just described. They are referred to here as runaway solutions.

The MM-MA system can be written as a set of evolution equations for the substrates, the substrate-enzyme complexes and the free enzymes. The right hand sides of the equations of the second and third types for a given choice of enzyme differ only by an overall sign. Adding them gives a conservation law for the total amount of enzyme $\rho_{\alpha}=x_{\mathrm{A}_{\mathrm{i}} \mathrm{E}_{\alpha}}+x_{\mathrm{E}_{\alpha}}$. The conservation laws can be used to eliminate the concentrations of the free enzymes from the evolution equations for the substrates and the substrate-enzyme complexes. The evolution equations for the free enzymes can be discarded. This leads to the system

$$
\begin{aligned}
& \left.\frac{d x_{\mathrm{A}_{\mathrm{m}}}}{d t}=-\sum_{\alpha: i(\alpha)=m} r(\alpha) C^{+}(\alpha) x_{\mathrm{A}_{\mathrm{i}(\alpha)}^{r(\alpha)}}^{r\left(\rho_{\alpha}\right.}-x_{\mathrm{A}_{\mathrm{i}(\alpha)} \mathrm{E}_{\alpha}}\right) \\
& +\sum_{\alpha: i(\alpha)=m} r(\alpha) C^{-}(\alpha) x_{\mathrm{A}_{\mathrm{i}(\alpha)} \mathrm{E}_{\alpha}}+\sum_{\alpha: f(\alpha)=m} s(\alpha) \Gamma(\alpha) x_{\mathrm{A}_{\mathrm{i}(\alpha)} \mathrm{E}_{\alpha}} \\
& \frac{d x_{\mathrm{A}_{\mathrm{i}(\alpha)}} \mathrm{E}_{\alpha}}{d t}=C^{+}(\alpha) x_{\mathrm{A}_{\mathrm{i}(\alpha)}(\alpha)}^{r(\alpha)}\left(\rho_{\alpha}-x_{\mathrm{A}_{\mathrm{i}(\alpha)} \mathrm{E}_{\alpha}}\right)-\left(C^{-}(\alpha)+\Gamma(\alpha)\right) x_{\mathrm{A}_{\mathrm{i}(\alpha)}} \mathrm{E}_{\alpha}
\end{aligned}
$$

Here $C^{+}(\alpha), C^{-}(\alpha)$ and $\Gamma(\alpha)$ are the reaction constants for the reactions involving the enzyme $E_{\alpha}$. The numbers $r(\alpha)$ and $s(\alpha)$ are the stoichiometric coefficients of the reaction catalysed by $E_{\alpha}$, referred to for brevity as the reaction $\alpha$. The number of molecules of substrate entering the reaction is $r(\alpha)$ and the number of molecules of product which result is denoted by $s(\alpha)$. In fact we allow $r(\alpha)$ and $s(\alpha)$ to be any real numbers satisfying the condition $r(\alpha) \geq 1$. This inequality ensures that the coefficients in the system of ODE are $C^{1} . i(\alpha)$ is the index labelling the substrate entering the reaction $\alpha$ and $f(\alpha)$ is the index labelling the product of that reaction. The full MM-MA system consists 
of (4.23), (4.24) and evolution equations for the concentrations of the free enzymes. Equations (4.23) and (4.24) are equivalent to the full MM-MA system in the following sense. If a solution of the full MM-MA system is given then the conserved quantities $\rho_{\alpha}$ can be computed. Then the concentrations of the substrates and the substrate-enzyme complexes satisfy (4.23) and (4.24) with those values of the $\rho_{\alpha}$. Conversely, suppose that a solution of (4.23) and (4.24) is given with certain values of the $\rho_{\alpha}$ and that the concentration of $x_{\mathrm{A}_{\mathrm{i}(\alpha)} \mathrm{E}_{\alpha}}$ is always less than $\rho_{\alpha}$. Then defining the concentrations of the free enzymes by $x_{\mathrm{E}_{\alpha}}=\rho_{\alpha}-x_{\mathrm{A}_{\mathrm{i}(\alpha)}} \mathrm{E}_{\alpha}$ gives a solution of the full MM-MA system. The following linear combination of equations (4.23) and (4.24) will be useful later.

$$
\begin{aligned}
& \frac{d}{d t}\left(x_{\mathrm{A}_{\mathrm{m}}}+\sum_{\alpha: i(\alpha)=m} r(\alpha) x_{\left.\mathrm{A}_{\mathrm{i}(\alpha)} \mathrm{E}_{\alpha}\right)} r(\alpha) \Gamma(\alpha) x_{\mathrm{A}_{\mathrm{i}(\alpha)} \mathrm{E}_{\alpha}-\sum_{\alpha: f(\alpha)=m} s(\alpha) \Gamma(\alpha) x_{\mathrm{A}_{\mathrm{i}(\alpha)} \mathrm{E}_{\alpha} .}} r \sum_{\alpha: i(\alpha)=m} r(\alpha)\right.
\end{aligned}
$$

In order to investigate when the MM-MA system admits runaway solutions a first step is to look for consistent leading order asymptotics. This is done using the following ansatz.

$$
\begin{aligned}
& x_{\mathrm{A}_{\mathrm{m}}}=\theta_{m} t+\ldots, \\
& x_{\mathrm{E}_{\alpha}}=\eta_{\alpha} t^{-r(\alpha)}+\ldots .
\end{aligned}
$$

For consistency $x_{\mathrm{A}_{\mathrm{i}(\alpha)} \mathrm{E}_{\alpha}}=\rho_{\alpha}-\eta_{\alpha} t^{-r(\alpha)}+\ldots$ These relations and their formal time derivatives are now inserted into the evolution equations. Comparing coefficients results in the equations

$$
\begin{aligned}
& \theta_{m}=-\sum_{\alpha: i(\alpha)=m} r(\alpha) C^{+}(\alpha) \theta_{i(\alpha)}^{r(\alpha)} \eta_{\alpha} \\
& +\sum_{\alpha: i(\alpha)=m} r(\alpha) C^{-}(\alpha) \rho_{\alpha}+\sum_{\alpha: f(\alpha)=m} \Gamma(\alpha) s(\alpha) \rho_{\alpha} \\
& 0=C^{+}(\alpha) \theta_{i(\alpha)}^{r(\alpha)} \eta_{\alpha}-\left(C^{-}(\alpha)+\Gamma(\alpha)\right) \rho_{\alpha} .
\end{aligned}
$$

Substituting the second equation into the first (or comparing coefficients in (4.25) gives

$$
\theta_{m}=-\sum_{\alpha: i(\alpha)=m} r(\alpha) \Gamma(\alpha) \rho_{\alpha}+\sum_{\alpha: f(\alpha)=m} s(\alpha) \Gamma(\alpha) \rho_{\alpha} .
$$

Since the $\theta_{m}$ are positive this implies a linear system of inequalities for the quantities $\rho_{\alpha}$. If these inequalities admit non-trivial solutions then the network is said to be autocatalytic. For a general network it is not easy to determine whether it is autocatalytic. The network of [9] modelling the Calvin cycle is easily shown to be autocatalytic. The network obtained by replacing the stoichiometric coefficients used in 9] by those used in [22] can be checked to be autocatalytic by 
an almost identical computation. When a network is autocatalytic and the $\rho_{\alpha}$ satisfy suitable inequalities then the constants $\theta_{m}$ are determined by equation (4.30) and the constants $\eta_{\alpha}$ are determined by equation (4.29).

In order to prove the existence of runaway solutions for autocatalytic MMMA systems it is convenient to introduce new variables adapted to the expected asymptotics. Define

$$
\begin{aligned}
& x_{\mathrm{A}_{\mathrm{m}}}(t)=Z_{m}(t)(t+R), \\
& x_{\mathrm{E}_{\alpha}}=\zeta_{\alpha}(t)(t+R)^{-r(\alpha)} .
\end{aligned}
$$

Then the solutions to be constructed should satisfy $Z_{m}(t) \rightarrow \theta_{m}$ and $\zeta_{\alpha}(t) \rightarrow \eta_{\alpha}$ as $t \rightarrow \infty$. The parameter $R \geq 1$ has been introduced to ensure that the leading terms in the quantities which tend to zero are already small for $t=0$. Rewriting the evolution equations in terms of the new variables leads to the system

$$
\begin{aligned}
& (t+R) \frac{d Z_{m}}{d t}+Z_{m}=-\sum_{\alpha: i(\alpha)=m} r(\alpha) C^{+}(\alpha) Z_{i(\alpha)}^{r(\alpha)} \zeta_{\alpha} \\
& +\sum_{\alpha: i(\alpha)=m} r(\alpha) C^{-}(\alpha) \rho_{\alpha}+\sum_{\alpha: f(\alpha)=m} s(\alpha) \Gamma(\alpha) \rho_{\alpha}-F_{m}\left(\zeta_{\alpha}\right) \\
& \frac{d \zeta_{\alpha}}{d t}+C^{+}(\alpha)(t+R)^{r(\alpha)} Z_{i(\alpha)}^{r(\alpha)} \zeta_{\alpha}=r(\alpha)(t+R)^{-1} \zeta_{\alpha} \\
& +(t+R)^{r(\alpha)}\left(C^{-}(\alpha)+\Gamma(\alpha)\right) \rho_{\alpha}-\left(C^{-}(\alpha)+\Gamma(\alpha)\right) \zeta_{\alpha}
\end{aligned}
$$

where

$$
\begin{aligned}
& F_{m}\left(\zeta_{\alpha}\right)=\sum_{\alpha: i(\alpha)=m} r(\alpha) C^{-}(\alpha) \zeta_{\alpha}(t+R)^{-r(\alpha)} \\
& +\sum_{\alpha: f(\alpha)=m} s(\alpha) \Gamma(\alpha) \zeta_{\alpha}(t+R)^{-r(\alpha)} .
\end{aligned}
$$

The main result is

Theorem 3 Let an autocatalytic reaction network be given. Then the corresponding MM-MA system can be written in the form (4.33)-(4.34) depending on a parameter $R$. Fix the values of the reaction constants. Define parameters $\theta_{m}$ and $\eta_{\alpha}$ by equations (4.30) and (4.29) and suppose that the $\theta_{m}$ are positive. Then there exist positive constants $K, R_{0}$ and $\delta_{0}$ such that if $R \geq R_{0}$ and

$$
\sum_{m}\left|Z_{m}(0)-\theta_{m}\right|+\sum_{\alpha}\left|\zeta_{\alpha}(0)-\eta_{\alpha}\right| \leq \delta_{0}
$$

then

$$
\sum_{m}\left|Z_{m}(t)-\theta_{m}\right|+\sum_{\alpha}\left|\zeta_{\alpha}(t)-\eta_{\alpha}\right| \leq K \delta_{0}
$$

for all $t \geq 0$ and

$$
\lim _{t \rightarrow \infty}\left(\sum_{m}\left|Z_{m}(t)-\theta_{m}\right|+\sum_{\alpha}\left|\zeta_{\alpha}(t)-\eta_{\alpha}\right|\right)=0 .
$$


To prove this theorem the first step is to rewrite the evolution equation for $\zeta_{\alpha}$ as an integral equation using variation of constants. Define

$$
\Psi_{\alpha}(s, t)=\exp \left[-\int_{s}^{t} C^{+}(\alpha)(u+R)^{r(\alpha)}\left(Z_{i(\alpha)}(u)\right)^{r(\alpha)} d u\right] .
$$

Here the fact that $\Psi_{\alpha}$ depends on $Z_{i(\alpha)}$ has not been made explicit in the notation. Then

$$
\begin{aligned}
\zeta_{\alpha}(t) & =\zeta_{\alpha}(0) \Psi_{\alpha}(0, t)+\int_{0}^{t} \Psi_{\alpha}(s, t)\left(C^{-}(\alpha)+\Gamma(\alpha)\right) \rho_{\alpha}(s+R)^{r(\alpha)} d s \\
& +\int_{0}^{t} \Psi_{\alpha}(s, t)\left[r(\alpha)(s+R)^{-1} \zeta_{\alpha}(s)-\left(C^{-}(\alpha)+\Gamma(\alpha)\right) \zeta_{\alpha}(s)\right] d s
\end{aligned}
$$

The second term on the right hand side of this equation can be transformed using the identity

$$
\frac{1}{C^{+}(\alpha)\left(Z_{i(\alpha)}(s)\right)^{r(\alpha)}} \frac{d}{d s}\left(\Psi_{\alpha}(s, t)\right)=(s+R)^{r(\alpha)} \Psi_{\alpha}(s, t)
$$

and integration by parts. The result is

$$
\begin{aligned}
\zeta_{\alpha}(t)- & \frac{\left(C^{-}(\alpha)+\Gamma(\alpha)\right) \rho_{\alpha}}{C^{+}(\alpha)\left(Z_{i(\alpha)}(t)\right)^{r(\alpha)}}=\left[\zeta_{\alpha}(0)-\frac{\left(C^{-}(\alpha)+\Gamma(\alpha)\right) \rho_{\alpha}}{C^{+}(\alpha)\left(Z_{i(\alpha)}(0)\right)^{r(\alpha)}}\right] \Psi_{\alpha}(0, t) \\
& +\int_{0}^{t} \Psi_{\alpha}(s, t) \frac{r(\alpha)\left(C^{-}(\alpha)+\Gamma(\alpha)\right) \rho_{\alpha}}{C^{+}(\alpha)\left(Z_{i(\alpha)}(s)\right)^{r(\alpha)+1}} \frac{d Z_{i(\alpha)}(s)}{d s} d s+\ldots
\end{aligned}
$$

where the last term in (4.39) has not been written out again.

Proof of Theorem $3 \mathrm{In}$ this proof it is assumed that $K$ and $R_{0}$ are greater than one. For positive constants $K$ and $\delta$ define

$$
t^{*}=\sup \left\{t>0: \sum_{m}\left|Z_{m}(t)-\theta_{m}\right|+\sum_{\alpha}\left|\zeta_{\alpha}(t)-\eta_{\alpha}\right| \leq 2 K \delta_{0}\right\} .
$$

Suppose that $\delta_{0} \leq \frac{1}{4} K^{-1} \min \left\{\theta_{m}\right\}$. This implies that the inequality $Z_{m} \geq \theta_{m} / 2$ holds on $\left[0, t^{*}\right]$. The time derivatives of the quantities $Z_{m}$ can be bounded by a constant $K$ depending only on the parameters in the system. To obtain estimates for $\Psi_{\alpha}$ the following auxiliary estimate is useful. For a positive constant 
a

$$
\begin{aligned}
& \int_{0}^{t} \exp \left[-a(r(\alpha)+1) \int_{s}^{t}(u+R)^{r(\alpha)} d u\right] d s \\
& =\exp \left[-a(t+R)^{r(\alpha)+1}\right] \int_{0}^{t} \exp \left[a(s+R)^{r(\alpha)+1}\right] d s \\
& =\exp \left[-a(t+R)^{r(\alpha)+1}\right] \int_{0}^{t} \frac{1}{a(r(\alpha)+1)(s+R)^{r(\alpha)}} \frac{d}{d s}\left(\exp \left[a(s+R)^{r(\alpha)+1}\right]\right) d s \\
& =\frac{1}{a(r(\alpha)+1)(t+R)^{r(\alpha)}}-\frac{\exp \left[a R^{r(\alpha)+1}-a(t+R)^{r(\alpha)+1}\right]}{a(r(\alpha)+1) R^{r(\alpha)}} \\
& +\int_{0}^{t} \frac{r(\alpha)}{a(r(\alpha)+1)(s+R)^{r(\alpha)}} \exp \left[a(s+R)^{r(\alpha)+1}-a(t+R)^{r(\alpha)+1}\right] d s .
\end{aligned}
$$

Choosing $R_{0}$ large enough ensures that the first factor in the last integral is smaller than $\frac{1}{2}$. Thus the integral term can be absorbed into the left hand side of the inequality. Discarding a term with a good sign gives

$$
\int_{0}^{t} \exp \left[-a(r(\alpha)+1) \int_{s}^{t}(u+R)^{r(\alpha)} d u\right] d s \leq \frac{2}{a(r(\alpha)+1)(t+R)^{r(\alpha)}} .
$$

Making a suitable choice of the constant $a$ leads to the inequality

$$
\int_{0}^{t} \Psi_{\alpha}(s, t) d s \leq K(t+R)^{-r(\alpha)}
$$

Putting this information into (4.41) gives

$$
\left|\zeta_{\alpha}(t)-\frac{\left(C^{-}(\alpha)+\Gamma(\alpha)\right) \rho_{\alpha}}{C^{+}(\alpha)\left(Z_{i(\alpha)}(t)\right)^{r(\alpha)}}\right| \leq\left[\zeta_{\alpha}(0)-\frac{\left(C^{-}(\alpha)+\Gamma(\alpha)\right) \rho_{\alpha}}{C^{+}(\alpha)\left(Z_{i(\alpha)}(0)\right)^{r(\alpha)}}\right]+K(t+R)^{-r(\alpha)} .
$$

The first term can be bounded using the smallness condition on the initial data and the second by using (4.45) and choosing $R_{0}$ large. It follows that for $R_{0}$ sufficiently large and $\delta_{0}$ sufficiently small

$$
\left|\zeta_{\alpha}(t)-\frac{\left(C^{-}(\alpha)+\Gamma(\alpha)\right) \rho_{\alpha}}{C^{+}(\alpha)\left(Z_{i(\alpha)}(t)\right)^{r(\alpha)}}\right| \leq \frac{K \delta_{0}}{2} .
$$

The evolution equation for $Z_{m}$ can be rewritten in the form

$$
\begin{aligned}
& (t+R) \frac{d Z_{m}}{d t}+\left(Z_{m}-\theta_{m}\right)= \\
& -\sum_{\alpha: i(\alpha)=m} r(\alpha) C^{+}(\alpha) Z_{i(\alpha)}^{r(\alpha)}\left[\zeta_{\alpha}-\frac{\left(C^{-}(\alpha)+\Gamma(\alpha)\right) \rho_{\alpha}}{C^{+}(\alpha) Z_{i(\alpha)}^{r(\alpha)}}\right] \\
& -F_{m}\left(\zeta_{\alpha}\right) .
\end{aligned}
$$


The right hand side of (4.48) can be bounded by $K \delta_{0} / 2$ after possibly increasing $K$ and $R_{0}$. Integrating this gives an inequality of the form

$$
(t+R) \sum_{m}\left|Z_{m}-\theta_{m}\right| \leq\left(K \delta_{0} / 2\right)(t+1)
$$

and hence

$$
\sum_{m}\left|Z_{m}-\theta_{m}\right| \leq \frac{K \delta_{0}}{2}
$$

Combining (4.50) and (4.47) gives

$$
\sum_{\alpha}\left|\zeta_{\alpha}(t)-\eta_{\alpha}\right| \leq \frac{K \delta_{0}}{2}
$$

It can be concluded that $t^{*}=\infty$ and the first part of the theorem is proved. The integrand in the definition of $\Psi_{\alpha}$ is bounded below by a positive constant and thus $\Psi_{\alpha}(0, t) \rightarrow 0$ as $t \rightarrow \infty$. Combining this with (4.41) shows that

$$
\zeta_{\alpha}(t)-\frac{\left(C^{-}(\alpha)+\Gamma(\alpha)\right) \rho_{\alpha}}{C^{+}(\alpha)\left(Z_{i(\alpha)}(t)\right)^{r(\alpha)}} \rightarrow 0
$$

as $t \rightarrow \infty$. It can then be concluded from (4.48) that $\frac{d}{d t}\left((t+R)\left(Z_{m}-\theta_{m}\right)\right)=o(1)$. Hence $(t+R)\left(Z_{m}-\theta_{m}\right)=o(t)$ and $Z_{m} \rightarrow \theta_{m}$ as $t \rightarrow \infty$. Together with the information we already have this implies that $\zeta_{\alpha}-\eta_{\alpha} \rightarrow 0$ as $t \rightarrow \infty$ and this completes the proof of the theorem.

Consider now stationary solutions of MM-MA. Equation (4.25) implies that the equation obtained by setting $\theta_{m}=0$ in (4.30) holds in the stationary case. This is a linear system for the substrate-enzyme complexes. It is a system of $n$ equations for $r$ unknowns. In the case of the system (4.1)-(4.12) there are five equations for seven unknowns and it is easily seen that the solution space is of dimension two. The equations are those obtained by setting the time derivatives to zero in (4.13). Explicitly

$$
\begin{aligned}
& x_{\mathrm{RuBPE}_{1}}=\frac{k_{15}}{k_{3}} x_{\mathrm{Ru}_{5 \mathrm{PE}_{5}}}, \\
& x_{\mathrm{PGAE}_{2}}=\frac{2 k_{3}}{k_{6}} x_{\mathrm{RuBPE}_{1}}-\frac{k_{18}}{k_{6}} x_{\mathrm{PGAE}_{6}}, \\
& x_{\mathrm{DPGAE}_{3}}=\frac{k_{6}}{k_{9}} x_{\mathrm{PGAE}_{2}}, \\
& x_{\mathrm{GAPE}_{4}}=\frac{k_{9}}{5 k_{12}} x_{\mathrm{DPGAE}_{3}}-\frac{k_{21}}{5 k_{12}} x_{\mathrm{GAPE}_{7}}, \\
& x_{\mathrm{Ru}_{5 \mathrm{PE}_{5}}}=\frac{3 k_{12}}{k_{15}} x_{\mathrm{GAPE}_{4}} .
\end{aligned}
$$

Suppose now that we prescribe the values of $x_{\mathrm{PGAE}_{6}}$ and $x_{\mathrm{GAPE}_{7}}$. It is possible to derive the equation

$$
x_{\mathrm{GAPE}_{4}}=\frac{1}{k_{12}}\left(k_{18} x_{\mathrm{PGAE}_{6}}+k_{21} x_{\mathrm{GAPE}_{7}}\right) .
$$


Substituting back into equations (4.53)-(4.55) and (4.57) gives:

$$
\begin{aligned}
x_{\mathrm{RuBPE}_{1}} & =\frac{3}{k_{3}}\left(k_{18} x_{\mathrm{PGAE}_{6}}+k_{21} x_{\mathrm{GAPE}_{7}}\right), \\
x_{\mathrm{PGAE}_{2}} & =\frac{1}{k_{6}}\left(5 k_{18} x_{\mathrm{PGAE}_{6}}+6 k_{21} x_{\mathrm{GAPE}_{7}}\right), \\
x_{\mathrm{DPGAE}_{3}} & =\frac{1}{k_{9}}\left(5 k_{18} x_{\mathrm{PGAE}_{6}}+6 k_{21} x_{\mathrm{GAPE}_{7}}\right), \\
x_{\mathrm{Ru} \mathrm{PE}_{5}} & =\frac{3}{k_{15}}\left(k_{18} x_{\mathrm{PGAE}_{6}}+k_{21} x_{\mathrm{GAPE}_{7}}\right) .
\end{aligned}
$$

These can then be used to obtain expressions for the concentrations of the free enzymes. For the total amount of any one of the enzymes is equal to the amount of the free enzyme plus the amount of it bound to its substrate. Now these expressions will be used to extract information from the time evolution equations for the free enzymes. For brevity let $X=x_{\mathrm{PGAE}_{6}}$ and $Y=x_{\mathrm{GAPE}_{7}}$. Then

$$
\begin{aligned}
x_{\mathrm{RuBP}} & =\frac{3\left(k_{2}+k_{3}\right)\left(k_{18} X+k_{21} Y\right)}{k_{1}\left(k_{3} \rho_{1}-3 k_{18} X-3 k_{21} Y\right)}, \\
x_{\mathrm{PGA}} & =\frac{\left(k_{5}+k_{6}\right)\left(5 k_{18} X+6 k_{21} Y\right)}{k_{4}\left(k_{6} \rho_{2}-5 k_{18} X-6 k_{21} Y\right)}, \\
x_{\mathrm{DPGA}} & =\frac{\left(k_{8}+k_{9}\right)\left(5 k_{18} X+6 k_{21} Y\right)}{k_{7}\left(k_{9} \rho_{3}-5 k_{18} X-6 k_{21} Y\right)}, \\
x_{\mathrm{GAP}} & =\left[\frac{\left(k_{11}+k_{12}\right)\left(k_{18} X+k_{21} Y\right)}{k_{10}\left(k_{12} \rho_{4}-k_{18} X-k_{21} Y\right)}\right]^{\frac{1}{5}}, \\
x_{\mathrm{Ru} 5 \mathrm{P}} & =\frac{3\left(k_{14}+k_{15}\right)\left(k_{18} X+k_{21} Y\right)}{k_{13}\left(k_{15} \rho_{5}-3 k_{18} X-3 k_{21} Y\right)} .
\end{aligned}
$$

The expressions obtained up to now suffice to determine all unknowns in terms of $X, Y$ and the conserved quantities $\rho_{\alpha}$. There are, however, two further equations which lead to consistency conditions. These are:

$$
\begin{aligned}
& x_{\mathrm{PGA}}=\frac{\left(k_{17}+k_{18}\right) X}{k_{16}\left(\rho_{6}-X\right)}, \\
& x_{\mathrm{GAP}}=\frac{\left(k_{20}+k_{21}\right) Y}{k_{19}\left(\rho_{7}-Y\right)} .
\end{aligned}
$$

Note that equations (4.63)-(4.69) only have positive solutions under the restrictions that $X$ and $Y$ satisfy the inequalities which ensure the positivity of the denominators of the right hand sides. Rearranging the equations (4.68) and (4.69) gives

$$
\begin{aligned}
X & =\frac{k_{16} \rho_{6} x_{\mathrm{PGA}}}{k_{17}+k_{18}+k_{16} x_{\mathrm{PGA}}}, \\
Y & =\frac{k_{19} \rho_{7} x_{\mathrm{GAP}}}{k_{20}+k_{21}+k_{19} x_{\mathrm{GAP}}} .
\end{aligned}
$$


Hence

$$
5 k_{18} X+6 k_{21} Y=\frac{5 k_{16} k_{18} \rho_{6} x_{\mathrm{PGA}}}{k_{17}+k_{18}+k_{16} x_{\mathrm{PGA}}}+\frac{6 k_{19} k_{21} \rho_{7} x_{\mathrm{GAP}}}{k_{20}+k_{21}+k_{19} x_{\mathrm{GAP}}}
$$

and

$$
k_{18} X+k_{21} Y=\frac{k_{16} k_{18} \rho_{6} x_{\mathrm{PGA}}}{k_{17}+k_{18}+k_{16} x_{\mathrm{PGA}}}+\frac{k_{19} k_{21} \rho_{7} x_{\mathrm{GAP}}}{k_{20}+k_{21}+k_{19} x_{\mathrm{GAP}}} .
$$

Substituting these relations into (4.64) and (4.66) gives equations of the form:

$$
\begin{aligned}
& x_{\mathrm{PGA}}-g_{1}\left(x_{\mathrm{PGA}}, x_{\mathrm{GAP}}\right)=0, \\
& x_{\mathrm{GAP}}^{5}-g_{2}\left(x_{\mathrm{PGA}}, x_{\mathrm{GAP}}\right)=0
\end{aligned}
$$

for some rational functions $g_{1}$ and $g_{2}$. More explicitly

$$
\begin{aligned}
& g_{1}\left(x_{\mathrm{PGA}}, x_{\mathrm{GAP}}\right)=\frac{a_{1} x_{\mathrm{PGA}}+a_{2} x_{\mathrm{GAP}}+a_{3} x_{\mathrm{PGA}} x_{\mathrm{GAP}}}{b_{1}+b_{2} x_{\mathrm{PGA}}+b_{3} x_{\mathrm{GAP}}+b_{4} x_{\mathrm{PGA}} x_{\mathrm{GAP}}}, \\
& g_{2}\left(x_{\mathrm{PGA}}, x_{\mathrm{GAP}}\right)=\frac{c_{1} x_{\mathrm{PGA}}+c_{2} x_{\mathrm{GAP}}+c_{3} x_{\mathrm{PGA}} x_{\mathrm{GAP}}}{d_{1}+d_{2} x_{\mathrm{PGA}}+d_{3} x_{\mathrm{GAP}}+d_{4} x_{\mathrm{PGA}} x_{\mathrm{GAP}}}
\end{aligned}
$$

for suitable constant coefficients depending on the $k_{i}$ and the $\rho_{\alpha}$.

Lemma 1 Any positive stationary solution of the system (4.1)-(4.12) defines a positive solution of the system (4.74)-(4.75). Conversely each positive solution of (4.74)-(4.75) with given values of $\rho_{\alpha}$ for which the quantities $X$ and $Y$ defined by (4.70) and (4.71) make the denominators in (4.63), 4.65) and (4.67) positive defines a positive stationary solution of the system (4.1)-(4.12).

Proof The first statement is a direct consequence of the calculations which have just been done. To prove the converse let $\left(x_{\mathrm{GAP}}, x_{\mathrm{PGA}}\right)$ be a solution of (4.74) - 4.75) and let $X$ and $Y$ be defined by (4.70) and (4.71). Then (4.68) and (4.69) are satisfied. It follows from (4.74)-(4.75) that (4.64) and (4.66) hold. Next define $x_{\mathrm{RuBP}}, x_{\mathrm{DPGA}}$ and $x_{\mathrm{Ru} 5 \mathrm{P}}$ by (4.63), (4.65) and (4.67) respectively. Define the quantities $x_{\mathrm{E}_{\mathrm{i}}}$ by the conservation laws and the quantities $x_{\mathrm{RuBPE}_{1}}$,

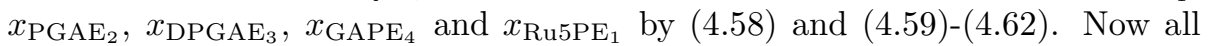
the variables in the system (4.1)-(4.12) have been defined and it remains to show that they define a stationary solution. Equations (4.58) and (4.59)-(4.62) imply (4.53)-(4.57). At this point it is useful to think of the system (4.1)-(4.12) as a special case of the MM-MA system introduced for a more general class of networks above. In that framework what has been obtained up to this point in the proof is a stationary solution of the equations (4.23), (4.24) and (4.25). It follows from the discussion above that this set of equations is equivalent to the full MM-MA system and this completes the proof of the lemma.

In 9 elementary flux modes of this system are investigated. This is a concept coming from chemical reaction network theory which can sometimes be used to investigate the number of stationary solutions of a dynamical system coming from a network of chemical reactions. To describe this in more detail it is necessary to introduce the notion of the deficiency of a reaction network. First 
note that a directed graph can be associated to any network where there is a vertex corresponding to each reaction complex and an arrow corresponding to each reaction. The connected components of this graph are called linkage classes. Let $n$ be the number of reaction complexes, $s$ the rank of the stoichiometric matrix and $l$ the number of linkage classes. Then the deficiency of the network is $\delta=n-s-l$. If the deficiency of the network (which is always non-negative) is equal to one and some other technical conditions are satisfied information about the number of stationary solutions can be obtained using what is called the deficiency one algorithm (D1A) [8]. There is a computer implementation of this algorithm which can be applied to cases where the network is not too large [6]. An elementary flux mode defines a subnetwork which is always of deficiency one 5]. Under suitable technical conditions stationary solutions of the subnetwork lead to corresponding stationary solutions of the full network and this can be proved using the implicit function theorem. In [9] this procedure is cited to conclude the existence of two distinct stationary solutions of the system MMMA in a given stoichiometric class. In what follows we will not say much more about this approach but the results obtained by using it were the starting point of the more direct proof of the existence of two stationary solutions given here.

In the MM-MA model for the Calvin cycle there are two stoichiometric generators and each defines a subnetwork. The subnetwork can be obtained by setting some of the reaction constants to zero. Here we procede directly using certain limits for the reaction constants corresponding to the two modes. Consider the system obtained from the system for stationary solutions of the system (4.1)-(4.12) by setting $k_{16}, k_{17}$ and $k_{18}$ to zero. Call it LS1. If the limiting values of the parameters are approached in such a way that $k_{17} / k_{16}$ and $k_{18} / k_{16}$ tend to non-zero limits $q_{17}$ and $q_{18}$ then the system varies in a way which is smooth up to the boundary. A similar system LS2 can be obtained by letting $k_{19}, k_{20}$ and $k_{21}$ tend to zero while $k_{20} / k_{19}$ and $k_{21} / k_{19}$ tend to non-zero limits $q_{20}$ and $q_{21}$.

Lemma 2 Consider the system LS1 and suppose that $k_{6} \rho_{2}-6 k_{21} \rho_{7} \geq 0$. If $k_{12} \rho_{4}-k_{21} \rho_{7} \geq 0$ then there is a unique positive solution. If $k_{12} \rho_{4}-k_{21} \rho_{7}<0$ then the number of solutions is zero, one or two according to whether

$$
\frac{1}{5}\left[\frac{4 k_{12} \rho_{4}\left(k_{20}+k_{21}\right)}{5 k_{19}\left(k_{12} \rho_{4}-k_{21} \rho_{7}\right)}\right]^{4} k_{10} \rho_{4}\left(k_{20}+k_{21}\right)-\left(k_{11}+k_{12}\right) k_{19} k_{21} \rho_{7}
$$

is negative, zero or positive, respectively.

Proof In this case the functions $g_{1}$ and $g_{2}$ in (4.74) and (4.75) only depend on $x_{\mathrm{GAP}}$. This means that (4.75) is an equation for $x_{\mathrm{GAP}}$ alone and for a suitable solution of this equation a corresponding value of $x_{\mathrm{PGA}}$ can be calculated. The explicit form of (4.75) is

$$
x_{\mathrm{GAP}}^{4}=\frac{\left(k_{11}+k_{12}\right) k_{19} k_{21} \rho_{7}}{k_{10} k_{12} \rho_{4}\left(k_{20}+k_{21}\right)+k_{10} k_{19}\left(k_{12} \rho_{4}-k_{21} \rho_{7}\right) x_{\mathrm{GAP}}} .
$$

When $k_{12} \rho_{4}-k_{21} \rho_{7} \geq 0$ the right hand side of this equation is non-increasing and the equation (4.79) has unique positive solution. The explicit form of (4.74) 
is

$$
x_{\mathrm{PGA}}=\frac{6\left(k_{5}+k_{6}\right) k_{19} k_{21} \rho_{7} x_{\mathrm{GAP}}}{k_{4} k_{6} \rho_{2}\left(k_{20}+k_{21}\right)+k_{4} k_{19}\left(k_{6} \rho_{2}-6 k_{21} \rho_{7}\right) x_{\mathrm{GAP}}} .
$$

If the right hand side of this is positive it defines an acceptable solution for $x_{\mathrm{GAP}}$ and the first statement of the lemma follows. Consider the case $k_{12} \rho_{4}-k_{21} \rho_{7}<$ 0 . Rewrite the equation schematically as

$$
x^{4}=\frac{\alpha}{\beta+\gamma x}
$$

where $\alpha>0, \beta>0$ and $\gamma<0$. If $p(x)=\gamma x^{5}+\beta x^{4}$ then the equation to be solved is $p(x)=\alpha$. The function $p$ has a unique maximum at $x_{*}=\frac{4 \beta}{-5 \gamma}$ and $p\left(x_{*}\right)=\frac{1}{5} \beta\left(\frac{4 \beta}{5 \gamma}\right)^{4}$. Comparing this quantity with $\alpha$ gives the second result of the lemma.

Lemma 3 Consider the system LS2 and suppose that $k_{12} \rho_{4}-k_{18} \rho_{6} \geq 0$. It has no positive solution if the quantities $k_{4} k_{6}\left(k_{17}+k_{18}\right) \rho_{2}-5\left(k_{5}+k_{6}\right) k_{16} k_{18} \rho_{6}$ and $-k_{6} \rho_{2}+5 k_{18} \rho_{6}$ are non-zero with opposite signs and exactly one positive solution when they are non-zero and have the same sign. The solution is given by

$$
\begin{aligned}
x_{\mathrm{PGA}} & =\frac{k_{4} k_{6}\left(k_{17}+k_{18}\right) \rho_{2}-5\left(k_{5}+k_{6}\right) k_{16} k_{18} \rho_{6}}{k_{4} k_{16}\left(-k_{6} \rho_{2}+5 k_{18} \rho_{6}\right)}, \\
x_{\mathrm{GAP}}^{5} & =\frac{\left(k_{11}+k_{12}\right) k_{16} k_{18} \rho_{6} x_{\mathrm{PGA}}}{k_{10} k_{12} \rho_{4}\left(k_{17}+k_{18}\right)+k_{10} k_{16}\left(k_{12} \rho_{4}-k_{18} \rho_{6}\right) x_{\mathrm{PGA}}} .
\end{aligned}
$$

The only other case where there exist positive solutions is when

$$
k_{4}\left(k_{17}+k_{18}\right)=\left(k_{5}+k_{6}\right) k_{16}
$$

and

$$
k_{6} \rho_{2}=5 k_{18} \rho_{6} .
$$

In that case $x_{\mathrm{PGA}}$ is arbitrary and there is a continuum of solutions.

Proof This is a direct calculation.

The parameters which are contained in $g_{1}$ and $g_{2}$ are

$$
k_{4}, k_{5}, k_{6}, k_{10}, k_{11}, k_{12}, k_{16}, k_{17}, k_{18}, k_{19}, k_{20}, k_{21}, \rho_{2}, \rho_{4}, \rho_{6}, \rho_{7}
$$

These equations do not depend on $\rho_{1}, \rho_{3}$ or $\rho_{5}$. Thus the conditions required to ensure the positivity of the solutions of (4.63), 4.65) and (4.67) can be guaranteed by choosing $\rho_{1}, \rho_{3}$ and $\rho_{5}$ large enough while keeping the other parameters fixed. In the two limiting cases considered above these functions simplify. In each limiting case the functions $g_{1}$ and $g_{2}$ depend on only one of the variables $x_{\mathrm{PGA}}$ and $x_{\mathrm{GAP}}$. Thus in that case one of the equations to be solved involves only one of the unknowns. If it can be solved then it can be substituted into the other equation to get the other variable. The derivative of the mapping sending the unknowns to the right hand side of the two equations is invertible 
in the two limiting cases. It follows by the implicit function theorem that for parameter sets close to those of the limiting cases the number of stationary solutions is independent of the values of the parameters. It follows in particular that there an open set in the space of parameters and conserved quantities for which this construction proves the existence of two distinct positive stationary solutions for given values of the parameters and conserved quantities.

It is possible to define a system MM-MAZ with mass action via MichaelisMenten kinetics starting from the data in 22. Like the other systems considered

up to now it has the property that $\bar{S}$ is positively invariant. In the case of the system MM-MAZ the quantities $\bar{x}_{i}$ satisfy evolution equations which are the same as those satisfied in the case of the system MM-MA except that the coefficients 5 and 3 are replaced by 1 and $\frac{3}{5}$. Next stationary solutions of MMMAZ will be considered. The concentrations of the substrate-enzyme complexes satisfy a system of linear equations similar to those in the case MM-MA. The relation between these linear systems can be expressed succinctly by saying that $k_{12}$ is replaced by $\frac{1}{5} k_{12}$. These linear equations can be solved for the concentrations of the first five complexes in terms of the other two. The result is similar to that for MM-MA with slightly different coefficients. In the equation for $x_{\mathrm{GAPE}_{4}}$ there is an extra factor of five while the equations for the other complexes are as before since they are independent of $k_{12}$. It is also possible to derive expressions for the concentrations of all substrates. These are all identical to the corresponding equations in the MM-MA case except for the equation for the concentration of GAP. This last equation is changed in two ways. The first is that $k_{12}$ is replaced by $\frac{1}{5} k_{12}$. The second is that the exponent $\frac{1}{5}$ is replaced by one. Equations similar to (4.74) and (4.75) can be derived, with the important difference that the fifth power in (4.75) is replaced by the first power. Thus (4.79) is replaced by a linear equation. This linear equation has a unique positive solution provided a certain sign condition is satisfied and no positive solution otherwise. Thus for the system MM-MAZ, in contrast to the system MM-MA, this construction does not lead to a proof of the existence of more than one stationary solution for any value of the parameters. Setting the right hand sides of the equivalents of equations (4.64) and (4.66) for the system MM-MA equal to the corresponding quantities coming from the right hand sides of equations (4.68) and (4.69) respectively shows that the set of $X$ and $Y$ defining stationary solution is the intersection of the zero set of two quadratic polynomials in $X$ and $Y$. Hence by Bézout's theorem [10] unless there is a continuum of solutions there are at most four.

\section{$5 \quad$ Michaelis-Menten kinetics}

Starting from the MM-MA system it is possible to obtain a simplified system, the Michaelis-Menten system (MM system) by passing to a singular limit. This will now be carried out formally. It will be convenient to describe this in the context of the more general system introduced in the last section. (The basic scheme is explained in a simpler case in Appendix A.) Let $\epsilon$ be a positive real 


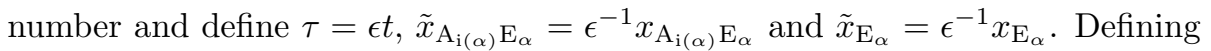
$\tilde{\rho}_{\alpha}=\epsilon^{-1} \rho_{\alpha}$ allows the transformation of the system to be carried out directly on the system (4.23)-(4.24). Equation (4.23) is identical to what it was originally except that the original quantities are replaced by the transformed quantities. The factors of $\epsilon$ cancel. On the other hand the equation (4.24) picks up an extra factor of $\epsilon$ on the left hand side. Formally taking the limit $\epsilon \rightarrow 0$ results in the equation obtained by setting the time derivative in (4.24) to zero. Solving this equation for $\tilde{x}_{\mathrm{A}_{\mathrm{i}(\alpha)} \mathrm{E}_{\alpha}}$ gives

$$
\tilde{x}_{\mathrm{A}_{\mathrm{i}(\alpha)} \mathrm{E}_{\alpha}}=\frac{C^{+}(\alpha) x_{\mathrm{A}_{\mathrm{i}(\alpha)}(\alpha)}^{r(\rho)} \tilde{\rho}_{\alpha}}{C^{+}(\alpha) x_{\mathrm{A}_{\mathrm{i}(\alpha)}^{r(\alpha)}}^{r(\alpha)}+C^{-}(\alpha)+\Gamma(\alpha)} .
$$

Substituting this back into equation (4.23) gives the MM system

$$
\begin{aligned}
& \frac{d x_{\mathrm{A}_{\mathrm{m}}}}{d \tau}=-\sum_{\alpha: i(\alpha)=m} \frac{C^{+}(\alpha) \tilde{\rho}_{\alpha} r(\alpha) \Gamma(\alpha) x_{\mathrm{A}_{\mathrm{i}(\alpha)}^{r(\alpha)}}^{C^{+}(\alpha) x_{\mathrm{A}_{\mathrm{i}(\alpha)}^{r(\alpha)}}^{((\alpha)}+C^{-}(\alpha)+\Gamma(\alpha)}}{+\sum_{\alpha: f(\alpha)=m} \frac{C^{+}(\alpha) \tilde{\rho}_{\alpha} s(\alpha) \Gamma(\alpha) x_{\mathrm{A}_{\mathrm{i}(\alpha)}^{r(\alpha)}}}{C^{+}(\alpha) x_{\mathrm{A}_{\mathrm{i}(\alpha)}^{r(\alpha)}}^{r(\alpha)}+C^{-}(\alpha)+\Gamma(\alpha)} .}
\end{aligned}
$$

Consider the ansatz $x_{\mathrm{A}_{\mathrm{m}}}=\tilde{\theta}_{m} \tau+\ldots$ for runaway solutions of (5.2). Substituting into the equation and comparing coefficients gives the equation obtained from (4.30) by replacing $\theta_{m}$ and $\rho_{\alpha}$ by $\tilde{\theta}_{m}$ and $\tilde{\rho}_{\alpha}$. Define a new variable $\tilde{Z}_{m}$ by

$$
x_{\mathrm{A}_{\mathrm{m}}}(\tau)=\tilde{Z}_{m}(\tau)(\tau+R) .
$$

Then equation (5.2) becomes

$$
\begin{aligned}
& (\tau+R) \frac{d \tilde{Z}_{m}}{d \tau}+\tilde{Z}_{m}= \\
& -\sum_{\alpha: i(\alpha)=m} \frac{\tilde{\rho}_{\alpha} r(\alpha) \Gamma(\alpha)}{1+\left(C^{+}(\alpha)\right)^{-1} \tilde{Z}_{i(\alpha)}^{-r(\alpha)}\left(C^{-}(\alpha)+\Gamma(\alpha)\right)(\tau+R)^{-r(\alpha)}} \\
& +\sum_{\alpha: f(\alpha)=m} \frac{\tilde{\rho}_{\alpha} s(\alpha) \Gamma(\alpha)}{1+\left(C^{+}(\alpha)\right)^{-1} \tilde{Z}_{i(\alpha)}^{-r(\alpha)}\left(C^{-}(\alpha)+\Gamma(\alpha)\right)(\tau+R)^{-r(\alpha)}} .
\end{aligned}
$$

Theorem 4 Let an autocatalytic reaction network be given. Then the corresponding MM system can be written in the form (5.2) depending on a parameter $R$. Fix the values of the reaction constants. The positive parameters $\tilde{\theta}_{m}$ are determined by the reaction constants. There exist positive constants $K, R_{0}$ and $\delta_{0}$ such that if $R \geq R_{0}$ and

$$
\sum_{m}\left|\tilde{Z}_{m}(0)-\tilde{\theta}_{m}\right| \leq \delta_{0}
$$


then

$$
\sum_{m}\left|\tilde{Z}_{m}(\tau)-\tilde{\theta}_{m}\right| \leq K \delta_{0}
$$

for all $\tau \geq 0$ and

$$
\lim _{\tau \rightarrow \infty} \sum_{m}\left|\tilde{Z}_{m}(\tau)-\tilde{\theta}_{m}\right|=0
$$

Proof The proof is similar to that of Theorem 3 but simpler. Define

$$
\tau^{*}=\sup \left\{\tau>0: \sum_{m}\left|\tilde{Z}_{m}(\tau)-\tilde{\theta}_{m}\right| \leq 2 K \delta_{0}\right\} .
$$

Choose $\delta_{0}$ small enough so that $\left|\tilde{Z}_{m}(\tau)\right| \geq \tilde{\theta} / 2$ on $\left[0, \tau^{*}\right]$. It follows from (5.4) that

$$
\left|(\tau+R) \frac{d \tilde{Z}_{m}}{d \tau}+\left(\tilde{Z}_{m}-\tilde{\theta}_{m}\right)\right| \leq K(\tau+R)^{-1} .
$$

Integrating this in time and choosing $R_{0}$ large enough gives an inequality of the form

$$
(\tau+R)\left(\tilde{Z}_{m}-\tilde{\theta}_{m}\right)(\tau) \leq K \delta_{0}(1+\tau)^{\epsilon}
$$

for any $\epsilon>0$. This allows the bootstrap assumption to be improved if $\tau^{*}$ is finite and it follows that in fact $\tau^{*}=\infty$. Using (5.9) again gives the final statement of the theorem.

Stationary solutions of the MM-MA system give rise to equations similar to those defined by the runaway solutions. In that case the analogue of (5.1) without tildes holds. Substituting this into the evolution equation for the substrates gives the equation (5.4) without tildes. This means that any stationary solution of the MM-MA system defines a stationary solution of the MM system. Conversely, any stationary solution of the MM system defines a stationary solution of the system (4.23)-(4.24). It was already shown that any solution of the latter system defined a solution of the system MM-MA and if the solution of (4.23)(4.24) is stationary the solution of the system MM-MA is so too. Thus there is a one to one correspondence between stationary solutions of the MM system and stationary solutions of the MM-MA system for fixed values of the conserved quantities $\rho_{\alpha}$. To get the standard form of the Michaelis-Menten system as used in 22 the numerators and denominators in all the summands on the right hand side should be divided by $C^{+}(\alpha)$. Hence if the MM system is given in isolation the reaction constants of the MM-MA system it comes from are not determined uniquely. Only the expressions $\Gamma(\alpha)$ and $\frac{C^{-}(\alpha)+\Gamma(\alpha)}{C^{+}(\alpha)}$ are determined.

Equations for a model with Michaelis-Menten kinetics are written in Appendix A of [22]. In fact, as has been remarked in [4, the expression for $v_{5}$ in 22 is not correct since it includes a dependence of the reaction on ATP, which does not agree with the reaction it is supposed to model, the sixth reaction in Table B2 of 22. Here we consider the correct model obtained from the reaction network given in 22 by using Michaelis-Menten kinetics and call it the model MMZ in what follows. The discrepancy just mentioned only affects 
the biological interpretation of the constants in the system studied here and not the general mathematical form of the equations. The variables used in 22 . include the concentration of ATP but this is assumed to be constant and so no evolution equation is required for it. A system with Michaelis-Menten kinetics is also considered (but not written explicitly) in [9] and is called MM in what follows. The only difference between these two systems is that the expression $v_{4}$ in 22] is replaced by an expression of the form $\frac{A x_{\mathrm{GAP}}^{5}}{B+x_{\mathrm{GAP}}^{5}}$ for some positive constants $A$ and $B$. For both systems the positive orthant $S$ is invariant. In these Michaelis-Menten systems the right hand sides of the evolution equations are bounded functions of their arguments and so solutions exist globally to the future. Making use of these facts it can be shown as in the case of the systems MA and MAZ that there are no $\omega$-limit points on the boundary except possibly the origin. To see this it is enough to use the structure of the first equation in Appendix A of [22].

In both cases

$$
\frac{d L_{1}}{d t}=-\frac{1}{2}\left(v_{5}-\frac{1}{5} v_{2}\right)-\frac{3}{5} v_{6}
$$

where the $v_{j}$ denote reaction rates. With the corrected value of $v_{5}$ we get

$$
v_{5}-\frac{1}{5} v_{2}=\frac{V_{5 \max } x_{\mathrm{PGA}}}{\left(x_{\mathrm{PGA}}+K_{m 5}\right)}-\frac{1}{5}\left[\frac{V_{2 \max } x_{\mathrm{PGA}} x_{\mathrm{ATP}}}{\left(x_{\mathrm{PGA}}+K_{m 21}\right)\left(x_{\mathrm{ATP}}+K_{m 22}\right)}\right] .
$$

The positivity of this is equivalent to the inequality

$$
\begin{aligned}
& \left(x_{\mathrm{PGA}}+K_{m 5}\right) V_{2 \max } x_{\mathrm{ATP}} \\
& \leq 5\left(x_{\mathrm{PGA}}+K_{m 21}\right)\left(x_{\mathrm{ATP}}+K_{m 22}\right) V_{5 \max } .
\end{aligned}
$$

This can be rewritten as

$$
x_{\mathrm{PGA}} \leq \frac{5 K_{m 21}\left(x_{\mathrm{ATP}}+K_{m 22}\right) V_{5 \max }-K_{m 5} V_{2 \max } x_{\mathrm{ATP}}}{V_{2 \max } x_{\mathrm{ATP}}-5\left(x_{\mathrm{ATP}}+K_{m 22}\right) V_{5 \max }}
$$

provided the denominator is non-zero. Call the right hand side of this equation $K$ and suppose that $K>0$. Then if a solution initially satisfies the inequality $\frac{5}{3} L_{1} \leq K$ it tends to zero as $t \rightarrow \infty$. For the values of the parameters given in Appendix B of [22] $K$ is about 0.14 .

\section{A model including a dynamical description of ATP}

In [9] a model with diffusion is considered which is given by the equations (13) of that reference. They define a system of reaction diffusion equations. This system is denoted by MAd. Setting the diffusion coefficient equal to zero (or restricting to spatially homogeneous solutions) gives a system of ODE different 
from (2.1)-(2.5) due to the inclusion of the concentration of ATP as a dynamical variable. Call this ODE system MAdh. The explicit form of this system is

$$
\begin{aligned}
& \frac{d x_{\mathrm{RuBP}}}{d t}=k_{5} x_{\mathrm{Ru} 5 \mathrm{P}} x_{\mathrm{ATP}}-k_{1} x_{\mathrm{RuBP}}, \\
& \frac{d x_{\mathrm{PGA}}}{d t}=2 k_{1} x_{\mathrm{RuBP}}-k_{2} x_{\mathrm{PGA}} x_{\mathrm{ATP}}-k_{6} x_{\mathrm{PGA}}, \\
& \frac{d x_{\mathrm{DPGA}}}{d t}=k_{2} x_{\mathrm{PGA}} x_{\mathrm{ATP}}-k_{3} x_{\mathrm{DPGA}}, \\
& \frac{d x_{\mathrm{GAP}}}{d t}=k_{3} x_{\mathrm{DPGA}}-5 k_{4} x_{\mathrm{GAP}}^{5}-k_{7} x_{\mathrm{GAP}}, \\
& \frac{d x_{\mathrm{Ru} 5 \mathrm{P}}}{d t}=-k_{5} x_{\mathrm{Ru} 5 \mathrm{P}} x_{\mathrm{ATP}}+3 k_{4} x_{\mathrm{GAP}}^{5}, \\
& \frac{d x_{\mathrm{ATP}}}{d t}=-k_{2} x_{\mathrm{PGA}} x_{\mathrm{ATP}}-k_{5} x_{\mathrm{Ru} 5 \mathrm{P}} x_{\mathrm{ATP}}+k_{8}\left(c-x_{\mathrm{ATP}}\right)
\end{aligned}
$$

for a constant $c$. Adding equations (6.1) and (6.6) gives

$$
\frac{d}{d t}\left(x_{\mathrm{RuBP}}+x_{\mathrm{ATP}}\right)=-k_{1} x_{\mathrm{RuBP}}-k_{2} x_{\mathrm{PGA}} x_{\mathrm{ATP}}+k_{8}\left(c-x_{\mathrm{ATP}}\right) .
$$

Let $m=\min \left\{k_{1}, k_{8}\right\}$. Then

$$
\frac{d}{d t}\left(x_{\mathrm{RuBP}}+x_{\mathrm{ATP}}\right) \leq-m\left(x_{\mathrm{RuBP}}+x_{\mathrm{ATP}}\right)+k_{8} c .
$$

It follows that $x_{\mathrm{RuBP}}+x_{\mathrm{ATP}}$ can be bounded by the maximum of its initial value and the quantity $\frac{k_{8} c}{m}$. Call this $\hat{x}_{\mathrm{RuBP}}$. The evolution equation for $x_{\mathrm{PGA}}$ implies that this quantity is bounded by the maximum of its initial value and $\hat{x}_{\mathrm{PGA}}=\frac{2 k_{1} \hat{x}_{\mathrm{RuBP}}}{k_{6}}$. Similarly the quantities $x_{\mathrm{DPGA}}$ and $x_{\mathrm{GAP}}$ are bounded by the maximum of their initial values and

$$
\hat{x}_{\mathrm{DPGA}}=\frac{k_{2} \hat{x}_{\mathrm{PGA}} \hat{x}_{\mathrm{RuBP}}}{k_{3}}, \quad \hat{x}_{\mathrm{GAP}}=\frac{k_{3} \hat{x}_{\mathrm{DPGA}}}{k_{7}},
$$

respectively.

Obtaining a bound for $x_{\mathrm{Ru} 5 \mathrm{P}}$ is more complicated. Integrating the evolution equation for $x_{\mathrm{DPGA}}$ on the interval $[s, t]$ and using the fact that $x_{\mathrm{PGA}}$ and $x_{\mathrm{DPGA}}$ are bounded leads to an inequality of the form

$$
\int_{s}^{t} x_{\mathrm{DPGA}}(\xi) d \xi \leq C\left(\int_{s}^{t} x_{\mathrm{ATP}}(\xi) d \xi+1\right)
$$

for a positive constant $C$. Similarly it follows from the evolution equation for $x_{\mathrm{GAP}}$ that

$$
\int_{s}^{t} x_{\mathrm{GAP}}(\xi) d \xi \leq C\left(\int_{s}^{t} x_{\mathrm{DPGA}}(\xi) d \xi+1\right) .
$$

Combining these two inequalities gives

$$
\int_{s}^{t} x_{\mathrm{GAP}}(\xi) d \xi \leq C\left(\int_{s}^{t} x_{\mathrm{ATP}}(\xi) d \xi+1\right) .
$$


By variation of constants the evolution equation for $x_{R u 5 P}$ implies

$$
\begin{aligned}
& x_{\mathrm{Ru} 5 \mathrm{P}}(t)=x_{\mathrm{Ru} 5 \mathrm{P}}(0) \exp \left(-k_{5} \int_{0}^{t} x_{\mathrm{ATP}}(s) d s\right) \\
& +3 k_{4} \int_{0}^{t} \exp \left(-k_{5} \int_{s}^{t} x_{\mathrm{ATP}}(\xi) d \xi\right)\left(x_{\mathrm{GAP}}(s)\right)^{5} d s .
\end{aligned}
$$

for a positive constant $C^{\prime}$. The first term is bounded and the second can be bounded by an expression of the form

$$
C \int_{0}^{t} \exp \left(-C^{\prime} \int_{s}^{t} x_{\mathrm{GAP}}(\xi) d \xi\right) x_{\mathrm{GAP}}(s) d s .
$$

The integrand in the last expression can be written in terms of the derivative of $\exp \left(-C^{\prime} \int_{s}^{t} x_{\mathrm{GAP}}(\xi) d \xi\right)$. Thus this expression can be bounded by

$$
C\left[1-\exp \left(-C^{\prime} \int_{0}^{t} x_{\mathrm{GAP}}(\xi) d \xi\right)\right] .
$$

It follows that $x_{\mathrm{Ru} 5 \mathrm{P}}$ is bounded.

Since solutions of the system MAdh are bounded they exist for all future times. If a solution of MAdh has an $\omega$-limit point on the boundary of $S$ then $x_{\text {ATP }}$ is positive there. It follows, using the same argument as was applied to the system MA, that if a solution of MAdh has an $\omega$-limit point on the boundary then all concentrations except that of ATP are zero there. The evolution equation for $x_{\mathrm{ATP}}$ then shows that $x_{\mathrm{ATP}}=c$ at that point. Linearizing about the point $(0,0,0,0,0, c)$ shows that it is a hyperbolic sink.

Consider the stationary solutions of MAdh, i.e. the homogeneous stationary solutions of the MAd model. Combining the first and fifth equations gives $x_{\mathrm{GAP}}^{5}=\left(k_{1} / 3 k_{4}\right) x_{\mathrm{RuBP}}$, just as in the system MA. In fact, as shown in [9], for a stationary solution all other concentrations can be expressed in terms of $x_{\mathrm{RuBP}}$. Note first that

$$
x_{\mathrm{ATP}}=c-\frac{8 k_{1} x_{\mathrm{RuBP}}}{3 k_{8}}-\frac{k_{7}}{k_{8}}\left(\frac{k_{1} x_{\mathrm{RuBP}}}{3 k_{4}}\right)^{\frac{1}{5}} .
$$

For an admissible solution the right hand of this equation must be positive. If this is satisfied the concentrations other than $x_{\mathrm{RuBP}}$ can be computed. The result is that $x_{\mathrm{PGA}}=\frac{2 k_{1} x_{\mathrm{RuBP}}}{k_{2} x_{\mathrm{ATP}}+k_{6}}, x_{\mathrm{DPGA}}=\frac{k_{7} x_{\mathrm{GAP}}}{k_{3}}+\frac{5 k_{4} x_{\mathrm{GAP}}^{5}}{k_{3}}, x_{\mathrm{Ru} 5 \mathrm{P}}=\frac{3 k_{4} x_{\mathrm{GAP}}^{5}}{k_{5} x_{\mathrm{ATP}}}$. Substituting all these relations into the evolution equation for PGA gives an equation for $x_{\mathrm{RuBP}}$ alone. It is of degree ten and hence it is not easy to extract information from it.

An alternative approach is the following. Another equation which can be derived for stationary solutions of MAdh is

$$
x_{\mathrm{GAP}}=\left[\frac{k_{7}\left(k_{2} x_{\mathrm{ATP}}+k_{6}\right)}{k_{4}\left(k_{2} x_{\mathrm{ATP}}-5 k_{6}\right)}\right]^{\frac{1}{4}} .
$$


On the other hand, the equation (6.16) can be rewritten as

$$
x_{\mathrm{ATP}}=c-\frac{8 k_{4}}{k_{8}} x_{\mathrm{GAP}}^{5}-\frac{k_{7}}{k_{8}} x_{\mathrm{GAP}} .
$$

Thus we have a set of two equations for the two quantities $x_{\mathrm{GAP}}$ and $x_{\mathrm{ATP}}$ and solving these is equivalent to determining all stationary solutions of MAdh. Write the equation (6.17) schematically as $x_{\mathrm{GAP}}=F_{1}\left(x_{\mathrm{ATP}}\right)$. The function $F_{1}$ is decreasing on the region $x_{\mathrm{ATP}}>5 k_{6} / k_{2}$ where it is real. Note that the right hand side of (6.18) is a decreasing function of $x_{\mathrm{GAP}}$ and thus this equation can be inverted to write it schematically as $x_{\mathrm{GAP}}=F_{2}\left(x_{\mathrm{ATP}}\right)$ for a decreasing function $F_{2}$ defined on the interval $[0, c]$ with $F_{2}(c)=0$. Stationary solutions of MAdh are in one to one correspondence with intersections of the graphs of $F_{1}$ and $F_{2}$. The function $F_{1}$ is strictly convex since $F_{1}^{\prime \prime}>0$. On the other hand, the function $F_{2}$ is strictly concave. It follows that the two graphs can intersect in at most two points for any given values of the parameters. If $c<5 k_{6} / k_{2}$ they do not intersect at all. For fixed values of the reaction constants if $c$ is sufficiently large the graphs intersect in two points.

For the system MAdh

$$
\frac{d L_{1}}{d t}=-\frac{1}{2}\left(k_{6}-\frac{1}{5} k_{2} x_{\mathrm{ATP}}\right) x_{\mathrm{PGA}}-\frac{3}{5} k_{7} x_{\mathrm{GAP}}
$$

Note that in this system $x_{\mathrm{ATP}}$ is bounded by $c$. Hence to make $L_{1}$ a Lyapunov function it suffices to require the inequality $c k_{2} \leq 5 k_{6}$. Thus when this inequality is satisfied all solutions converge to the origin as $t \rightarrow \infty$. For the system MAdh the function $L_{2}$ with $\alpha=\frac{1}{2}$ satisfies the same equation as it does for the system MA. Hence the same conclusion can be drawn about solutions which converge to zero.

One remark will be made on the behaviour of solutions of systems including a diffusion term. In fact we can do this in any space dimension, adding diffusion terms in any subset of the equations with any choice of positive diffusion constants. Suppose that the domain of the spatial variables is a bounded region $\Omega$ with smooth boundary and assume that Neumann boundary conditions hold. Let $\mathcal{L}_{1}=\int_{\Omega} L_{1}$. Then

$$
\frac{d \mathcal{L}_{1}}{d t}=-\int_{\Omega}\left[\frac{1}{2}\left(k_{6}-\frac{1}{5} k_{2} x_{\mathrm{ATP}}\right) x_{\mathrm{PGA}}-\frac{3}{5} k_{7} x_{\mathrm{GAP}}\right] .
$$

When the inequality $k_{6}-\frac{1}{5} k_{2} x_{\mathrm{ATP}} \geq 0$ holds $\mathcal{L}_{1}$ is a Lyapunov function. In particular, there are no stationary solutions, homogeneous or inhomogeneous, when $c k_{2} \leq 5 k_{6}$.

\section{Conclusions}

An important motivation for the work of [22] and [9] on models of the Calvin cycle was to see if photosynthesis can work in different steady states. This led to 
the question of whether the relevant mathematical models admit more than one stable positive stationary solution. For related work see also [21, [4, [13] and [14. It was already shown in [9] that a simple model using mass action kinetics (the model called MA) never admits even one solution of this type. Depending on the values of the reaction constants, either there is no positive stationary solution at all or if there is it is unstable. This suggests that this is not a very good model. Trying to understand more about what actually happens in this model leads to the mathematical question of what the solutions departing from a small neighbourhood of the stationary solution actually do or, more generally, what the long-time behaviour of solutions is. Theorem 1 of this paper provides partial answers to this question. When there is no stationary solution the concentrations of all substrates tend to zero. When there is a stationary solution some solutions have the property that all concentrations tend to zero while others have the property that all concentrations tend to infinity (runaway solutions). The latter alternative seems to be a further undesirable property of the model.

The model MM-MA, which has a much larger number of unknowns, does admit more than one stationary solution for certain values of the reaction constants, as was shown in [9], and numerical results indicate that one of them is stable. In this paper this existence result was reproduced by a more direct method. The model MM has the same stationary solutions as the model MM-MA and thus also admits two stationary solutions for certain choices of parameters. Applying the same method to the related system MMZ with the stoichiometric coefficients taken from 22 does not reveal the presence of multiple stationary solutions and it may be that in that case there are none. From this point of view the models MM-MA and MM look better that the model MA but in fact, as shown in Theorems 3 and 4 of this paper, both the models MM-MA and MM exhibit runaway solutions. An intuitive explanation for the existence of these solutions is that in all these models the concentration of ATP, which is the energy source for the reactions, is not modelled explicitly. Instead ATP is implicitly assumed to be plentiful and thus present at a constant level. In [9] another model is considered where diffusion is taken into account and the concentration of ATP is modelled dynamically. Restricting to spatially homogeneous solutions leads to a system of ODE called MAdh. Interestingly, we were able to show here that all solutions of MAdh remain bounded, so that there are no runaway solutions in that model. Although heuristically plausible this is subtle to prove. For suitable values of the parameters this system also admits two positive stationary solutions.

There seem to be few general results available on the boundedness of solutions of systems of ODE arising from chemical reaction networks with mass action kinetics. One theorem says, using the language of Chemical Reaction Network Theory, that in a mass action system which is weakly reversible and has only one linkage class all solutions are bounded [2]. Neither of the main hypotheses of that result hold for any of the systems considered in this paper but perhaps some of the techniques used there might be adapted to give information about models for the Calvin cycle. For weakly reversible systems it might be 
possible to prove that solutions do not have $\omega$-limit points on the boundary of the positive orthant. Information about this and relevant references can be found in 3 .

It is of interest to compare the conditions which allow the conclusions of the theorems in this paper to be obtained with values of the parameters which are biologically reasonable. To do this we start from the biological data collected in Appendix B of 22]. In that paper values are given relating to Michaelis-Menten kinetics. Assuming that Michaelis-Menten kinetics goes over into mass action kinetics when the concentration of the substrate is small compared to that of the enzyme it is possible to get values for the reaction constant in equations (2.1)-2.5). The results are

$$
\begin{aligned}
& \left(\frac{V_{1 \max }}{K_{m 1}}, \frac{V_{2 \max } x_{\mathrm{ATP}}}{K_{m 21} K_{m 22}}, \frac{V_{3 \max }}{K_{m 3}}, \frac{V_{4 \max }}{K_{m 4}}, \frac{V_{5 \max }}{K_{m 5}}, \frac{V_{6 \max }}{K_{m 6}}, \frac{V_{13 \max } x_{\mathrm{ATP}}}{K_{m 131} K_{m 132}}\right) \\
& =(3.78,125,10.1,9.63,4,0.02,4.52) .
\end{aligned}
$$

With these values of the reaction constants the ratio $\frac{k_{2}}{5 k_{6}}$ which plays a key role in Theorem 1 is equal to 1250 . Thus these values are well within the regime where a positive stationary solution exists.

Is it true that for the system MA with $k_{2}>5 k_{6}$ every solution either tends to infinity, the origin or the positive stationary solution? This is not known but since (2.1)-(2.5) is what is called a monotone cyclic feedback system it follows from the main theorem of [15] that bounded solutions have $\omega$-limit sets which are no worse that those of a two-dimensional system. Using similar ideas it can be shown that almost all bounded solutions converge to the stationary solution. The system (2.1)-(2.5) satisfies $\frac{\partial f_{i}}{\partial x_{i}} \geq 0$ for $i \neq j$ and is thus cooperative. It is also irreducible in the sense that no non-trivial coordinate hyperplane is left invariant by the Jacobian at any point. Using this the result on convergence of all bounded solutions except for those whose initial conditions belong to a set of measure zero follows from a theorem of Hirsch [11].

In this paper a variety of different results have been proved about the dynamics of solutions of mathematical models for the Calvin cycle. A number of interesting open questions remain to be investigated. It would be desirable to have rigorous results on stability of the stationary solutions and the (non)existence of periodic solutions. Inhomogeneous solutions of the system with diffusion should be investigated following the initial work in 9. It would be good to extend the results of this paper to more general models of the Calvin cycle such as those of [17] and [18]. Finally, the basic motivating question remains: are there mathematical models of the Calvin cycle where it can be proved that there are at least two homogeneous stable positive stationary solutions?

\section{A Michaelis-Menten theory}

Consider a simple reaction which converts one molecule of the species $S$ (the substrate) to one molecule of the substance $P$ (the product). With mass action 
kinetics this leads to the equations $\dot{x}_{S}=-k x_{S}$ and $\dot{x}_{P}=k x_{S}$. Suppose now that this reaction is catalysed by an enzyme $E$. Then there is a reaction with reaction constant $k_{1}$ in which the substrate combines with the enzyme to form a complex $S E$. The reaction constant for the process of dissociation of $S E$ into $S$ and $E$ will be denoted by $k_{-1}$. Finally there is the reaction in which the complex gives rise to the product with reaction constant $k_{2}$ while setting free the enzyme. This gives rise to the system

$$
\begin{aligned}
\dot{x}_{S} & =-k_{1} x_{S} x_{E}+k_{-1} x_{S E}, \\
\dot{x}_{S E} & =k_{1} x_{S} x_{E}-\left(k_{-1}+k_{2}\right) x_{S E}, \\
\dot{x}_{E} & =-k_{1} x_{S} x_{E}+\left(k_{-1}+k_{2}\right) x_{S E}, \\
\dot{x}_{P} & =k_{2} x_{S E} .
\end{aligned}
$$

The first three of these equations form a closed system and thus it is natural to analyse it first and use the last equation to determine the evolution of the concentration of the product afterwards, if desired. The above system is the MM-MA version of the original simple reaction. The Michaelis-Menten kinetics will now be derived on a heuristic level. Note first that the quantity $x_{S E}+x_{E}$ is conserved. Call it $E_{0}$. Substituting the relation $x_{E}=E_{0}-x_{S E}$ into the first two evolution equations gives a closed system for $x_{S}$ and $x_{S E}$ :

$$
\begin{aligned}
\dot{x}_{S} & =-k_{1} E_{0} x_{S}+\left(k_{-1}+k_{1} x_{S}\right) x_{S E}, \\
\dot{x}_{S E} & =k_{1} E_{0} x_{S}-\left(k_{-1}+k_{1} x_{S}+k_{2}\right) x_{S E} .
\end{aligned}
$$

Now introduce $\tau=\epsilon t, \tilde{x}_{S E}=\epsilon^{-1} x_{S E}$ and $\tilde{E}_{0}=\epsilon^{-1} E_{0}$ for a constant $\epsilon$. This gives

$$
\begin{aligned}
x_{S}^{\prime} & =-k_{1} \tilde{E}_{0} x_{S}+\left(k_{-1}+k_{1} x_{S}\right) \tilde{x}_{S E} \\
\epsilon \tilde{x}_{S E}^{\prime} & =k_{1} \tilde{E}_{0} x_{S}-\left(k_{-1}+k_{1} x_{S}+k_{2}\right) \tilde{x}_{S E}
\end{aligned}
$$

where the primes denote derivative with respect to $\tau$. In the last system it is possibly to formally pass to the limit $\epsilon \rightarrow 0$, corresponding to a very small amount of enzyme. In the limit the second equation reduces to the algebraic equation

$$
\tilde{x}_{S E}=\frac{k_{1} \tilde{E}_{0} x_{S}}{k_{-1}+k_{1} x_{S}+k_{2}} .
$$

Substituting this back into the evolution equation for $x_{S}$ and gives the effective Michaelis-Menten equation

$$
x_{S}^{\prime}=-\frac{k_{1} k_{2} \tilde{E}_{0} x_{S}}{k_{1} x_{S}+k_{-1}+k_{2}} .
$$

It can then be computed that in this set-up $x_{P}^{\prime}=-x_{S}^{\prime}$.

This type of discussion is quite standard and the reason it is reproduced here is to illuminate the relations between the three types of kinetics (MA, MM-MA and MM) by an explanation of the simplest example. There is a one to one 
correspondence between stationary solutions of the systems MM-MA and MM, as will now be shown. If a stationary solution $\left(x_{S}, x_{S E}\right)$ of the system MM-MA is given then the equation (A.9) is satisfied. Hence the equation (A.10) holds and a stationary solution of the system MM is obtained. Conversely, suppose that a solution $\left(x_{S}, \tilde{x}_{S E}\right)$ is given. Then a stationary solution of the system A.5 is obtained. Defining $x_{E}=E_{0}-x_{S E}$ for a positive constant $E_{0}$ completes it to a stationary solution of the system MM-MA.

\section{B A special class of matrices}

This appendix is concerned with the algebraic properties of some matrices of a special form which appear in this paper. Let $A$ be an $n \times n$ matrix with entries $a_{i j}$. Suppose that $a_{i i}<0$ for each $i$, that $a_{i j}>0$ for $j=i-1 \bmod n$ and that $a_{i j}=0$ otherwise. Suppose further that $(-1)^{n+1} \operatorname{det} A>0$. The matrix $A+\lambda I$ is positive for a sufficiently large real number $\lambda$, i.e all its elements are positive. By the Perron-Frobenius theorem [16 it has a unique eigendirection spanned by a positive vector. Let $p$ be an eigenvector of this type with components $p_{i}$. The corresponding eigenvalue is positive. Let it be denoted by $\beta$. Another consequence of the Perron-Frobenius theorem is that all other eigenvalues of $A+\lambda I$ have modulus smaller than $\beta$. In particular the real part of any other eigenvalue is smaller than $\beta$. The vector $p$ is an eigenvector of $A$ with eigenvalue $\alpha=\beta-\lambda$ and all other eigenvalues of $A$ have real part smaller than $\alpha$.

If $A$ is a matrix of the above special form then it can be shown that the matrix $B=A^{-1}$ is a positive matrix. One way of proving this as follows. Let $x$ be a vector in $\mathbf{R}^{n}$ and consider the equation $A x=y$. Inverting the matrix is equivalent to solving this equation for $x$. The equation can be written in components as

$$
a_{i i} x_{i}+a_{i, i-1} x_{i-1}=y_{i} ; 1 \leq i \leq n
$$

where the indices are to be interpreted modulo $n$. Hence

$$
a_{i+1, i} x_{i}=\left(-a_{i+1, i+1}\right) x_{i+1}+y_{i+1} .
$$

Note that the coefficients in this equation are positive. By substituting these equations into each other successively with $i$ increasing from one to $n$ it is possible to obtain an equation of the form:

$$
\left(\prod_{i} a_{i, i+1}\right) x_{1}=\left(\prod_{i}\left(-a_{i i}\right)\right) x_{n}+\sum c_{i} y_{i}
$$

where the coefficients $c_{i}$ are positive. Rearranging this gives

$$
(-1)^{n+1}(\operatorname{det} A) x_{n}=\sum c_{i} y_{i}
$$

Any other $x_{i}$ can be determined in an analogous way. The determinant of $A$ is equal to $\prod_{i} a_{i i}+(-1)^{n+1} \prod_{i} a_{i, i+1}$. Putting these facts together gives the proof of the desired statement. 
It can be concluded from the Perron-Frobenius theorem that $B$ has a unique eigendirection spanned by a positive vector. This is also an eigenvector of $A$ and so must be proportional to $p$. The corresponding eigenvalue is $\alpha^{-1}$ and is positive. Hence $\alpha$ is positive.

Acknowledgements One of the authors (ADR) is grateful to Zoran Nikoloski for arousing his interest in this subject and for helpful discussions. This research was partially supported by the Hausdorff Center for Mathematics of the University of Bonn.

\section{References}

[1] Alberts, B., Johnson, A., Lewis, J., Raff, M., Roberts, K. and Watson, J. D. 2002 Molecular biology of the cell. Garland Science, New York.

[2] Anderson, D. A. 2011 Boundedness of trajectories for weakly reversible, single linkage class reaction systems. J. Math. Chem. 49, 2275-2290.

[3] Anderson, D. A. 2011 A proof of the Global Attractor Conjecture in the single linkage class case. SIAM J. Appl. Math. 71, 1487-1508.

[4] Arnold, A. and Nikoloski, Z. 2011 A quantitative comparison of CalvinBenson cycle models. Trends in Plant Science 16, 676-682.

[5] Conradi, C., Flockerzi, D., Raisch, J. and Stelling, J. 2007 Subnetwork analysis reveals dynamical features of complex (bio)chemical networks. Proc. Natl. Acad. Sci. USA 104, 19175-19180.

[6] Ellison, P. and Feinberg, M. 2000 How catalytic mechanisms reveal themselves in multiple steady-state data. J. Mol. Catal. A: Chem. 154, 155-167.

[7] Feinberg, M. 1980 Lectures on chemical reaction networks. Available at http://www.chbmeng.ohio-state.edu/ feinberg/research/

[8] Feinberg, M. 1988 Chemical reaction network structure and the stability of complex isothermal reactors. II Multiple steady states for networks of deficiency one. Chem. Eng. Sci. 43, 1-25.

[9] Grimbs, S., Arnold, A., Koseska, A., Kurths, J., Selbig, J. and Nikoloski, Z. 2011 Spatiotemporal dynamics of the Calvin cycle: multistationarity and symmetry breaking instabilities. Biosystems 103, 212-223.

[10] Hartshorne, R. 1977 Algebraic geometry. Springer, Berlin.

[11] Hirsch, M. W. 1985 Systems of differential equations which are competitive or cooperative II: convergence almost everywhere. SIAM J. Math. Anal. 16, 432-439. 
[12] Huang, C.-Y. F. and Ferrell, J. E. 1996 Ultrasensitivity in the mitogenactivated protein kinase cascade. Proc. Natl. Acad. Sci. USA 93, 1007810083.

[13] Jablonsky, J., Bauwe, H. and Wolkenhauer, O. 2011 Modeling the CalvinBenson cycle. BMC Syst. Biol. 5, 185.

[14] Lei, H.-B., Wang, X., Wang, R., Chen, L. and Zhang, J.-F. 2011 A parameter condition for ruling out multiple equilibria of the photosynthetic carbon metabolism. Asian J. Control 13, 611-624.

[15] Mallet-Paret, J. and Smith, H. L. 1990 The Poincaré-Bendixson theorem for monotone cyclic feedback systems. J. Dyn. Diff. Eq. 2, 367-421.

[16] Meyer, C. D. 2000 Matrix analysis and applied linear algebra. SIAM, Philadelphia.

[17] Pettersson, G. and Ryde-Pettersson, U. 1988 A mathematical model of the Calvin photosynthesis cycle. Eur. J. Biochem. 175, 661-672.

[18] Poolman, M. G., Olcer, H., Lloyd, J. C., Raines, C. A. and Fell, D. 2001 Computer modelling and experimental evidence for two steady states in the photosynthetic Calvin cycle. Eur. J. Biochem. 268, 2810-2816.

[19] Rendall, A. D. 2012 Mathematics of the NFAT signalling pathway. SIAM J. Appl. Dyn. Sys. 11, 988-1006.

[20] Wang, L. and Sontag. E. D. 2008 On the number of steady states in a multiple futile cycle. J. Math. Biol. 57, 29-52.

[21] Zhu, X.-G., de Sturler, E. and Long, S. P. 2007 Optimizing the distribution of resources between enzymes of carbon metabolism can dramatically increase photosynthetic rate: a numerical simulation using an evolutionary algorithm. Plant Physiol. 145, 513-526.

[22] Zhu, X.-G., Alba, R. and de Sturler, E. 2009 A simple model of the Calvin cycle has only one physiologically feasible steady state under the same external conditions. Nonlin. Anal. RWA 10, 1490-1499. 